\title{
Cardinal Bayesian Allocation Mechanisms without Transfers
}

\author{
Antonio Miralles* \\ Universitat Autònoma de Barcelona \\ First version: January 2008. This version: December 2010
}

\begin{abstract}
In allocating goods with no use of monetary transfers, random allocation mechanisms can be designed in order to elicit information on preference intensities. I study the nontransfer allocation of two ex-ante identical objects under Bayesian incentive compatibility, with symmetric agents and independent private valuations. I find the ex-ante utilitarian-optimal mechanism, in which the probability of receiving a specified object is used as "numeraire" to purchase probability units of the other object. I characterize this mechanism as an appropriate combination of lotteries, auctions and insurance. The latter element ensures that efficient auctions are feasible. If the problem is constrained to guarantee exactly one object per agent, then the optimal mechanism uses no more information than the agents' ordinal preferences.
\end{abstract}

Keywords: Nontransfer Mechanism Design; Allocation; Private Information.

JEL codes: D02, D78, D82.

\section{Introduction}

An important question in Economics is how we should allocate our goods and resources. I study the allocation of two ex-ante identical objects to an arbitrary number of ex-ante identical agents, ${ }^{1}$ each possibly demanding

*antonio.miralles@uab.cat. Unitat de Fonaments de l'Anàlisi Econòmica, Departament d'Economia i Història Econòmica, Campus Bellaterra, Universitat Autònoma de Barcelona, 08193 Bellaterra, Barcelona, Spain. I am grateful to the anonymous referees, Atila Abdulkadiroğlu, Miguel Angel Ballester, Yeon-Koo Che, Barton Lipman, Zvika Neeman, Andrew F. Newman, Parag Pathak, Gregory Pavlov and Pedro ReyBiel for previous comments and suggestions. During the research I received financial support from the Caja Madrid Foundation, the Spanish Ministry of Science and Innovation (ECO2009-06946 and ECO200804756), Boston University and the European University Institute (Max Weber Programme), which I kindly acknowledge. Special thanks to Chiara.

${ }^{1}$ In this paper, ex-ante is understood as the stage at which the agents have not learnt their own preferences, although they know how these are stochastically distributed. Interim refers to the stage at which each agent 
both objects. In the allocation problem I study, each agent knows how she valuates these objects though she does not know the other agents' valuations. I solve the problem of finding a mechanism that allocates the objects so as to maximize ex-ante utilitarian welfare, i.e. the unweighted sum of every agent's $e x$ ante expected payoff, ${ }^{2}$ given the constraint that the mechanism cannot use any monetary transfer. To my knowledge, this is the first time that a mechanism design approach is taken in order to obtain a welfaremaximizing several-object random allocation mechanism without transfers.

When monetary transfers are allowed, the well-known Vickrey-Clarke-Groves mechanism (Vickrey, 1961; Clarke, 1971; Groves, 1973) uses these transfers to elicit information on each agent's preferences. ${ }^{3}$ Yet money is not used in many contexts, such as collusion in markets, task allocation in organizations, supply of company slots in airport terminals, children placement in public schools, housing allocation in colleges... This motivates the search for a solution to the allocation problem without monetary transfers.

The use of random allocations, or the allocation of assignment probabilities, proves to be useful in solving this problem. It exploits the agents' cardinal preferences, i.e. the intensity by which one object is preferred to the other, in a way that resembles a mechanism with monetary transfers. Intuitively, the assignment probability regarding one of the objects can play the role of money, i.e. it becomes the numeraire.

I find that, under certain plausible conditions on the distribution of valuations (more on this below), the optimal mechanism consists of a mixture of lotteries, auctions and insurance. When not all the agents declare to prefer the same good, each good is assigned by even lottery among the agents who declare a preference for it. When all the agents declare to prefer the same good, the non-preferred object is efficiently ${ }^{4}$ auctioned off using the allocation probabilities of the preferred object as the numeraire. Since this numeraire is scarce and the optimal unrestricted bid might be capped by the endowed probabilities of the preferred object, an insurance policy is needed for the object to be efficiently auctioned off. The insurance policy allows for lower bids than otherwise necessary to guarantee an efficient auction in exchange of a lower payoff in states of the world when an auction is not needed.

For the same reason the efficient auction alone may not provide the optimal random allocation, splitting the two objects in equal allocation probabilities among the agents and letting them trade these probabilities has only learnt her own preferences, although she knows how the other agents' preferences are distributed. In the ex-post stage, each agent has learnt all agents' preferences.

${ }^{2}$ This will be regarded simply as "welfare" throughout this paper. As noted by the referees, Harsanyi (1955) shows that any social welfare function takes the form of a weighted sum of individuals' cardinal utilities under simple axioms. If the "veil of ignorance" (no agent knows in advance which social position and its associated weight- she shall obtain, and she attaches the same subjective probability to every position) were imposed, all social positions would have the same weight in the welfare function. This would justify the utilitarian welfare approach.

${ }^{3}$ More recent work has analyzed the validity of the VCG mechanism under more general assumptions such as interdependent valuations (see for instance McLean and Postlewaite, 2006).

${ }^{4}$ The non-preferred object is assigned with probability 1 to the agent that relatively prefers it most. 
may not provide the optimal random allocation either. An agent could be willing to trade away more probability units of an object than what the given endowment allows for. Nevertheless, with only two agents, I show that no insurance policy is necessary in the equilibrium (the symmetric Bayesian Nash equilibrium, here as in the rest of this paper) of the game the mechanism induces. Both the market with equal endowments and the previous lottery-auction mechanism (without insurance) are optimal with two agents.

The steps in finding the solution to this allocation problem are as follows. Under a minor regularity condition, ${ }^{5}$ Proposition 1 tells us that if not all agents prefer the same object, an agent who prefers one of the objects shall not hold any chances to obtain her non-preferred object. In such a case, each object has to be randomly assigned among the agents who prefer it. In fact, an allocation that does not satisfy this condition would be dominated (in terms of welfare) by an allocation induced by an alternative mechanism ${ }^{6}$ that gives any agent more chances to obtain the object she prefers. The question then is how to proceed when all the agents prefer the same object.

This question could be understood as an efficient (welfare-maximizing) auction problem, in which the nonpreferred object is auctioned off using the allocation probabilities of the preferred object as the numeraire. In our problem, however, the numeraire generates a marginal utility to each agent that depends on her preferences, instead of being constant. In an efficient auction, the contribution that each agent makes to overall welfare given her preferences is simply her valuation for the auctioned object. The preference for the numeraire does not matter since it is the same for all agents. In the problem at hand, on the contrary, an agent's contribution to welfare, which I call criterion function, is rather more complex due to the difference just mentioned.

Additionally, the numeraire in our problem is a scarce good in itself. Proposition 2 simplifies this issue: if the criterion function is a non-negative function, we can ignore the constraints regarding the fact that the numeraire is scarce. A rough intuition is that in equilibrium, for each additional probability unit of obtaining the preferred object any agent must be willing to sacrifice more than one probability unit of the non-preferred object. Otherwise the agents would trade all the chances to obtain the non-preferred object away. The probability allocations of the non-preferred object are then extreme (i.e. more concentrated on a few agents) as compared to those of the preferred object. Thus if the non-preferred object is given away (which optimally happens when the criterion function is non-negative), allocating valid probabilities, the optimal mechanism would also generate valid allocation probabilities for the preferred object in equilibrium.

\footnotetext{
${ }^{5}$ This regularity condition is mathematically described in the main section, and it is met in very intituitive scenarios such as: 1) all cases in which each agent's expected valuation for an object, conditional on the agent's preference intensity for it, is nondecreasing in this preference intensity, 2) all cases in which if any two agents prefer the same object, and conditional on their preference intensities, both expected valuations for the preferred object exceed both expected valuations for the non-preferred object.

${ }^{6}$ That allocation is the outcome of the equilibrium of the game induced by the mechanism.
} 
Furthermore, the Corollary to Proposition 2 observes than when each agent's criterion function is (decreasingly) monotone in the intensity with which she prefers her preferred object over her non-preferred object, $^{7}$ the non-preferred object shall be optimally assigned to the agent with highest criterion function, that is, the agent that relatively prefers the non-preferred object most (an efficient auction). Hence, Propositions 1 and 2 and this Corollary (under the previous regularity and monotonicity conditions) ${ }^{8}$ brings us the procedure to implement the optimal mechanism that has been described: lotteries if not all agents prefer the same object, efficient auctions otherwise and insurance to guarantee that the efficient auction is feasible.

I also consider an extended allocation problem where there is an additional constraint that each agent receives exactly one object. Only unit demands are accepted. This constraint turns the problem into what I call the two-school random assignment problem. The additional constraint reduces the scope of the planner's design since assigning to an agent an additional probability unit regarding one object must come at the cost of reducing exactly one probability unit regarding the other object. This means that no truthful and useful information on preference intensities can be elicited, since the opportunity cost of an additional probability unit regarding an object must be the same for all agents. Consequently, in this two-object case, only ordinal preferences can be usefully elicited.

\section{Literature}

The literature has extensively analyzed nontransfer allocation mechanisms that rely on agents' ordinal preferences. This has typically been the case in one-sided and two-sided matching problems, in the social choice literature and in allocation games where more than one object might be allocated to a single agent (Gibbard, 1973; Satterthwaite, 1975; Olson, 1991; Pápai, 2000; Pápai, 2001; Ehlers and Klaus, 2003).

The ranking mechanism (Campbell, 1998; Pesendorfer, 2000) is appealing among these mechanisms in that: 1) as the number of objects grows large, ranking mechanism approaches full efficiency, and 2) under the i.i.d. assumption the ranking mechanism maximizes welfare among mechanisms using only information on ordinal preferences. The ranking mechanism works as follows with two objects. First, each agent declares which object she prefers. Then, the planner randomly allocates each object to an agent who declares a preference for it. If all agents declare to prefer the same object, each object is randomly allocated among all agents.

The present paper analyzes mechanisms where also the intensity of preferences is taken into account. In states of the world in which all agents prefer the same object, the optimal mechanism manages to efficiently assign the non-preferred object, whereas the ranking mechanism assigns probabilities evenly. Numerical comparisons between the optimal mechanism and the ranking mechanism reveal substantial welfare gains

\footnotetext{
${ }^{7}$ By comparison with auction theory, this condition would be analogous to the standard assumption that each agent's "virtual valuation" is monotonically increasing in her valuation for the auctioned object.

${ }^{8}$ Examples of well-known prior distributions of valuations that satisfy both the regularity and the monotone criterion function conditions are the uniform and the exponential distribution.
} 
with two agents. To illustrate the comparison, let us normalize the welfare attained when the planner is perfectly informed about the agents' preferences to 1 and the welfare under even randomization to 0 . Then, under uniform priors, the ranking mechanism obtains a welfare of $1 / 2$ whereas the optimal mechanism attains $2 / 3$. However, the welfare difference between the optimal mechanism and the ranking mechanism is of the order of $1 / 2^{n-1}$, where $n$ is the number of agents, vanishing when $n$ is infinitely large.

Many attempts to propose nontransfer mechanisms that account for the intensity of preferences are observed. A seminal work is Hylland and Zeckhauser (1979) on pseudomarkets. The mechanism designer, or planner, provides each agent with "fake" money (e.g. points). A pseudomarket for objects (job positions in their study) is generated in which agents buy units of probability to be assigned to each object using this fake money. A tatônement process yields equilibrium prices. The solution is naturally Pareto-efficient. The only drawback of this solution is that when the number of agents is finite, any agent might behave strategically in order to profitably alter equilibrium prices. Mechanisms accounting for the intensity of preferences are also studied in recent literature on voting and political compromise (Saari, 2000; Casella, 2005; Casella and Gelman, 2005; Börgers and Postl, 2006). Recent School Choice literature (studying the assignment of children to public schools) is also taking the intensity of preferences into account. For instance, Abdulkadiroğlu, Che and Yasuda (2009) argue that the Boston Mechanism, critisized since it is not strategy-proof, could perform better (in terms of welfare) than strategy-proof mechanisms such as Deferred Acceptance precisely because strategic agents indirectly submit information on their preference intensities to the assignment algorithm. Another related paper on nontransfer allocation with preference intensities is Abdulkadiroğlu and Loertscher (2007) on housing assignment. Finally, Condorelli (2010) has a very recent work in which a set of identical goods is optimally allocated using waiting time instead of money to elicit agents' preferences.

Departing from previous work, the present paper aims to find a welfare-maximizing random allocation mechanism in which agents interact strategically, each one only knowing her own valuations. I consider the two-object case for tractability reasons. But even the two-object case is challenging without transfers ${ }^{9}$ and involves two-dimensional private information. ${ }^{10}$ The derivation of the optimal allocation of more than two objects remains a subject for further research.

Section 2 presents and solves the optimal allocation problem. Section 3 deals with implementation and compares the optimal mechanism to the ranking mechanism. Section 4 analyzes the two-school random assignment problem. Section 5 discusses possible extensions. Section 6 concludes. An Appendix presents long proofs.

\footnotetext{
${ }^{9}$ Armstrong (2000) studies the assignment of two objects when monetary transfers can be used.

${ }^{10} \mathrm{I}$ avoid simplifications such as the use of unidimensional types to depict valuations for several objects (Krishna and Rosenthal, 1996; Levin, 1997; Menezes and Monteiro, 2003).
} 


\section{The optimal mechanism}

This Section formalizes and solves the nontransfer allocation problem. The problem is initially presented as a direct mechanism, where the random allocation is a function of the agents' reported preferences, and the allocation function is designed so that the agents have no incentives to misreport their preferences. Each agent's assigned probabilities depend on the other agents' reported preferences. However, the other agents' preferences are unknown to the former agent (the so-called independent private valuations assumption), thus what is relevant to her is the interim expected allocation, i.e. taking expectations of the assigned probabilities with respect to the distribution of the other agents' preferences. Then the problem is rewritten in its reduced form, where the random allocation mechanism is expressed in interim probabilities.

The second subsection solves this modified problem. The reduced-form, direct mechanism is useful because there is a standard procedure to simplify the problem that uses interim probabilities. The constraints implied by the need to refrain agents from misreporting their preferences, called the incentive compatibility constraints, are manipulated in order to get the interim allocation of one object as a function of the interim allocation of the other object. Propositions 1 and 2 characterize the way in which this simplified problem can be solved.

\subsection{The direct mechanism problem and its reduced form}

Two indivisible objects are to be allocated among $n$ agents. Each agent $i \in N \equiv\{1, \ldots, n\}$ has a nonnegative valuation $v_{h}^{i}$ for each object $h \in\{1,2\}$, measuring the utility that agent $i$ would obtain if she is given object $h$. Let $v^{i} \equiv\left(v_{1}^{i}, v_{2}^{i}\right)$. Each valuation vector is independently drawn from the same atomless distribution function $F$, with associated density $f$ which is assumed to be differentiable a.e.. For any positive valuations $x$ and $y$, assume that $f(x, y)=f(y, x)$. Therefore, the marginal distributions (denoted as $\tilde{F}$ throughout the paper), i.e. the distributions of the valuations for each object, are identical. ${ }^{11}$

Each agent $i$ learns the realization of her valuation vector $v^{i}$ only, according to the independent private valuations assumption. The planner only knows the prior distribution from which all valuations are drawn. The utility of an agent who obtains the two objects equals the sum of her valuations. For the moment, no restriction is placed on the number of objects an agent can obtain: zero, one or both. It might also be the case that some object is not given to any agent. Alternative assumptions are analyzed in Sections 4 and 5 .

The direct mechanism problem. The mechanism designer, or planner, designs a lottery mechanism to allocate the objects with the aim of maximizing the sum of the agents' ex-ante expected utilities. According to the Revelation Principle, the mechanism can be expressed as a direct mechanism, consisting of an ex-post probability matrix function $\tilde{q}: \mathbb{R}_{+}^{2 n} \rightarrow[0,1]^{2 n}$ subject to the feasibility constraints $\sum_{i=1}^{n} \tilde{q}_{h}^{i}\left(v^{1}, \ldots, v^{n}\right) \leq 1$,

\footnotetext{
${ }^{11}$ Note that this includes i.i.d. object valuation distributions.
} 
$\forall h \in\{1,2\}$, and to incentive compatibility (described below).

The reduced form, or interim, allocation probabilities are $\tilde{Q}_{h}^{i}\left(v^{i}\right) \equiv E_{N \backslash\{i\}} \tilde{q}_{h}^{i}\left(v^{1}, \ldots, v^{n}\right),{ }^{12}$ denoting $\tilde{Q}^{i}\left(v^{i}\right) \equiv\left(\tilde{Q}_{1}^{i}\left(v^{i}\right), \tilde{Q}_{2}^{i}\left(v^{i}\right)\right)$. Agent $i$ 's interim expected utility from the allocation mechanism is $\tilde{U}^{i}\left(v^{i}\right) \equiv$ $\tilde{Q}^{i}\left(v^{i}\right) \cdot v^{i}$. The objective function can be formulated as $\tilde{O} \equiv E_{N} \sum_{i=1}^{n} \tilde{U}^{i}\left(v^{i}\right)=E_{N} \sum_{i=1}^{n} \tilde{Q}^{i}\left(v^{i}\right) \cdot v^{i}$, i.e. the sum across agents of their ex-ante expected payoffs.

The (Bayesian, or interim) incentive compatibility constraints can be described as a set of inequalities $\tilde{Q}^{i}\left(v^{i}\right) \cdot v^{i} \geq \tilde{Q}^{i}\left(\tilde{v}^{i}\right) \cdot v^{i}, \forall i \in\{1, \ldots, n\}, \forall\left(v^{i}, \tilde{v}^{i}\right) \in \mathbb{R}_{+}^{4}$. That is, no agent has an incentive to misreport her preferences, given that no other agent misreports her own.

For each agent $i$, it is useful to transform her valuation vector into two new variables: a bundle valuation $B^{i}=v_{1}^{i}+v_{2}^{i}$, and a relative preference (or preference intensity) parameter $r^{i}=v_{2}^{i} / B^{i} \in[0,1] \cdot{ }^{13} B^{i}$ is the utility agent $i$ would obtain if she were given both objects and $r^{i}$ measures how intensely agent $i$ prefers object 2 to object 1 . Observe that $r^{i} \geq 1 / 2 \Longleftrightarrow v_{2}^{i} \geq v_{1}^{i}$ and that $r^{i}=1 / 2$ is equivalent to being indifferent between the two objects. Informally, agent $i$ has "intense preferences" when $\left|r^{i}-1 / 2\right|$ is "high", and "moderate preferences" when $\left|r^{i}-1 / 2\right|$ is "low".

There is a one-to-one relation between the relative preference parameter $r^{i}$ and the marginal rate of substitution $v_{2}^{i} / v_{1}^{i}$. Let $\Psi$ denote the distribution function of any $r^{i}$. Its associated density function is denoted by $\psi$. From my assumptions on $F$, the distribution of $r^{i}$ is symmetric with respect to $r=1 / 2$, i.e. $\psi\left(1-r^{i}\right)=\psi\left(r^{i}\right), \Psi\left(1-r^{i}\right)=1-\Psi\left(r^{i}\right)$, and $\Psi(1 / 2)=1 / 2$. The following lemma presents several simplifications of the problem. I omit the easy proof. ${ }^{14}$

Lemma 1 1) Any interim incentive compatible mechanism for this problem satisfies $\tilde{Q}^{i}\left(v^{i}\right) \cdot v^{i}=\tilde{Q}^{i}\left(a v^{i}\right) \cdot v^{i}$, for any $i, v^{i}$ and $a>0$ (Pesendorfer, 2000). Thus the planner can restrict herself to allocation functions that depend on relative preference parameters, namely $q:[0,1]^{n} \rightarrow[0,1]^{2 n}$.

2) Since agents are ex-ante symmetric, the planner can without loss of generality restrict attention to anonymous mechanisms, that is, for any $\left(r^{1}, \ldots, r^{n}\right) \in[0,1]^{n}$, any object $h$ and any permutation $\pi$ over an $n$-dimensional vector, we have $q_{h}\left(\pi\left(r^{1}, \ldots, r^{n}\right)\right)=\pi\left(q_{h}\left(r^{1}, \ldots, r^{n}\right)\right)$. An r-type agent's interim allocation probabilities can be denoted as $Q(r)=\left[Q_{1}(r), Q_{2}(r)\right]$, which does not depend on her identity.

3) Given the assumptions on $F$, the planner can without loss of generality restrict attention to allocation functions satisfying $q_{h}^{i}\left(r^{1}, \ldots, r^{n}\right)=q_{3-h}^{i}\left(1-r^{1}, \ldots, 1-r^{n}\right), \forall i \in\{1, \ldots, n\}, \forall h \in\{1,2\}$, a.e., and therefore $Q_{h}(r)=Q_{3-h}(1-r)$ a.e..

${ }^{12}$ Let $\tilde{N} \subseteq N$. Thoughout this paper, $E_{\tilde{N}}$ denotes the expectation operator taking the distribution of the preferences of all agents in $\tilde{N}$. If no subscript is used, $E$ denotes the expectation using the distribution $F$ of one agent's preferences.

${ }^{13}$ Armstrong (2000) uses polar coordinates instead, when dealing with the two-object revenue-maximizing auction design problem. His approach and the one taken here are equivalent.

${ }^{14}$ This lemma holds also for the case of strategy-proof mechanisms, as Hortalà-Vallvé (2007) shows. 
The anonymity of the optimal mechanism allows to skip the superscript identifying a specific agent $i$ (e.g. I use $r$ instead of $r^{i}$ and $v_{1}$ instead of $v_{1}^{i}$ ). In what follows $r, \tilde{r}$ and $r^{\prime}$ refer to different values of the relative preference parameter, and $\hat{r}$ denotes the relative preference parameter when treated as a random variable. For a given variable $X, E(X \mid r)$ denotes the conditional expectation of $X$, using the distribution $F$ of one agent's preferences, given $\hat{r}=r$.

Rewriting the problem in its reduced form. An agent's interim expected utility, given the rescaled vector $(B, r)$, is defined as $U(B, r) \equiv B\left[r Q_{2}(r)+(1-r) Q_{1}(r)\right]$. Since agents are ex-ante symmetric and all have the same weight in the welfare function, the planner can consider one agent's payoff as her objective function. This function can be expressed as $\int_{0}^{1} \psi(r) E(B \mid r) u(r) d r$, where

$$
u(r) \equiv r Q_{2}(r)+(1-r) Q_{1}(r)
$$

Due to the assumptions on $F$ and to the symmetry of the optimal mechanism, it can be shown that $E(B \mid r)=E(B \mid 1-r)$ and $u(r)=u(1-r)$, for any $r \in[0,1]$. Additionally, for any such $r$ it was already known that $\psi(r)=\psi(1-r)$. Under these circumstances, it is enough to maximize the following function,

$$
O \equiv \int_{1 / 2}^{1} \psi(r) E(B \mid r) u(r) d r
$$

and the optimal reduced-form probabilities need to be calculated only for $r \geq 1 / 2$.

The incentive compatibility constraints are rewritten as

$$
u(r) \equiv r Q_{2}(r)+(1-r) Q_{1}(r) \geq r Q_{2}(\tilde{r})+(1-r) Q_{1}(\tilde{r}), \forall(r, \tilde{r}) \in[0,1]^{2}
$$

Notice that $B$ disappears from these constraints, which is intuitive since $Q(\cdot)$ does not depend on the bundle valuation.

Remark 1 (IC) can be additionally simplified in that it just needs to apply to any pair $(r, \tilde{r}) \in[1 / 2,1]^{2}$.

Proof. Consider $r>1 / 2$ and $\tilde{r}<1 / 2$. (IC) jointly with the symmetry of $Q$ implies $Q_{2}(r) \geq Q_{1}(r)$ (otherwise, given $Q_{2}(1-r)=Q_{1}(r)$ and $Q_{1}(1-r)=Q_{2}(r)$ by the symmetry of $Q$, an $r$-type agent would profitably claim to be of type $1-r)$. Likewise, $Q_{2}(1-\tilde{r})=Q_{1}(\tilde{r}) \geq Q_{1}(1-\tilde{r})=Q_{2}(\tilde{r})$. Let us assume that (IC) is met for the pair $(r, 1-\tilde{r}) \in[1 / 2,1]^{2}$. Since $r>1-r$, we have $u(r) \geq r Q_{2}(1-\tilde{r})+(1-r) Q_{1}(1-\tilde{r}) \geq$ $r Q_{2}(\tilde{r})+(1-r) Q_{1}(\tilde{r})$, thus (IC) is met for $(r, \tilde{r})$. Let us assume that (IC) is met for the pair $(1-\tilde{r}, r) \in[1 / 2,1]^{2}$. Since $\tilde{r}<1-\tilde{r}$, we have $\tilde{r} Q_{2}(r)+(1-\tilde{r}) Q_{1}(r) \leq(1-\tilde{r}) Q_{2}(r)+\tilde{r} Q_{1}(r)(\leq u(1-\tilde{r}))$. By symmetry $u(\tilde{r})=u(1-\tilde{r})$, thus $(\mathrm{IC})$ is met for $(\tilde{r}, r)$. 
For the case in which both $r<1 / 2$ and $\tilde{r}<1 / 2$, the symmetry of $Q$ suffices to guarantee that (IC) is met for both $(r, \tilde{r})$ and the inverted pair $(\tilde{r}, r)$, provided it is met by both $(1-r, 1-\tilde{r})$ and the inverted pair $(1-\tilde{r}, 1-r)$.

Both the objective function and the incentive compatibility constraints depend on reduced-form (or interim) probabilities, whereas any feasibility constraints are typically characterized as a relation among ex-post allocation probabilities (where superscripts are still needed):

$$
\begin{aligned}
q_{h}^{i}\left(r^{1}, \ldots, r^{n}\right) & \geq 0, \forall i \in\{1, \ldots, n\}, \forall h \in\{1,2\} \\
\sum_{i=1}^{n} q_{h}^{i}\left(r^{1}, \ldots, r^{n}\right) & \leq 1, \forall h \in\{1,2\}
\end{aligned}
$$

Considering a reduced-form allocation $Q$, it is understood that it is feasible if there exists a feasible $e x$ post allocation $q$ such that for any $i \in N, E_{N \backslash\{i\}} q^{i}=Q$. The following result, adapted from Border (1991), allows for a restatement of feasibility constraints as a set of constraints affecting interim probabilities:

Lemma 2 Under the assumption that $Q_{2}(\cdot)$ is nondecreasing (equivalently, $Q_{1}(\cdot)$ is nonincreasing), a reducedform allocation $Q$ is feasible if and only if:

$$
\begin{array}{rlr}
R_{2}(r) \equiv 1-\Psi(r)^{n}-n \int_{r}^{1} \psi(x) Q_{2}(x) d x \geq 0 & & \left(Q_{2} \text {-constraints }\right) \\
R_{1}(r) \equiv & 1-[1-\Psi(r)]^{n} & \\
& -n \int_{1 / 2}^{1} \psi(x) Q_{2}(x) d x-n \int_{1 / 2}^{r} \psi(x) Q_{1}(x) d x & \\
\geq & 0 & \left(Q_{1}\right. \text {-constraints) }
\end{array}
$$

for any $r \in[1 / 2,1]$, with $Q_{2}(r) \geq 0$ and $Q_{1}(r) \geq 0$ for any such $r$.

Proof. From Border (1991), proposition 3.2, it is known that the feasibility constraints affecting object 2 are equivalent to the family of constraints $1-\Psi(r)^{n}-n \int_{r}^{1} \psi(x) Q_{2}(x) d x \geq 0$ for any $r \in[0,1]$, if $Q_{2}(\cdot)$ is nondecreasing and nonnegative. This obtains the $Q_{2}$-constraints when $r \geq 1 / 2$.

I construct the $Q_{1}-$ constraints taking values $\tilde{r} \leq 1 / 2$ and using $r=1-\tilde{r}: R_{2}(\tilde{r})=1-\Psi(\tilde{r})^{n}-$ $n \int_{\tilde{r}}^{1} \psi(x) Q_{2}(x) d x$ is equal to $R_{1}(r)=1-[1-\Psi(r)]^{n}-n \int_{1 / 2}^{1} \psi(x) Q_{2}(x) d x-n \int_{1 / 2}^{r} \psi(x) Q_{1}(x) d x$, by means of both the symmetry of the distribution of $r$ and the fact that $Q_{1}(r)=Q_{2}(1-r)$ a.e..

Given the symmetry in $Q$, feasibility with respect to object 2 implies feasibility with respect to object 1 , thus we can safely ignore the feasibility constraints related to object 1 .

Border's result is very useful in this setup. The reduced-form problem constructed provides manageable tools to prove proposition 1 in this paper. Additionally, in many auction problems an initial interim utility value (e.g. the interim expected utility of the agent with the lowest possible valuation) is obtained through 
optimality conditions or budget balance constraints. In the present paper, proposition 1 and a $Q_{2}$-constraint will be used to obtain such an initial value. Ex-post probabilities $q(\cdot)$ are not useful until the initial value problem is solved.

To sum up, the direct mechanism has been rewritten in its reduced form: it consists of an interim probability vector function $Q=\left(Q_{1}, Q_{2}\right):[1 / 2,1] \rightarrow[0,1]^{2}$ satisfying the family of constraints (IC), $\left(Q_{1}\right.$-constraints)

and $\left(Q_{2}\right.$-constraints). The objective function to maximize is $O \equiv \int_{1 / 2}^{1} \psi(r) E(B \mid r) u(r) d r=\int_{1 / 2}^{1} \psi(r) E(B \mid r)$. $\cdot\left[r Q_{2}(r)+(1-r) Q_{1}(r)\right] d r$.

\subsection{Solution}

As seen in the previous subsection, the optimal allocation mechanism can be solved as a reduced-form mechanism, in which optimal probabilities are calculated at the interim level. Although the restatement of the problem as a reduced-form symmetric mechanism has greatly reduced its complexity, the problem is still cumbersome. The solution process is ordered into several stages: 1) use (IC) to solve $Q_{2}(\cdot)$ out in terms of $Q_{1}(\cdot)$ and $\left.Q_{2}(1) ; 2\right)$ show that Border's feasibility constraints must bind at $1 / 2$, allowing me to solve $Q_{2}(1)$ out; and 3) show that the maximization problem can safely ignore the $Q_{2}$-constraints, so that the optimal $Q_{1}(\cdot)$ is solved for as in an efficient auction problem.

Solving $Q_{2}(\cdot)$ out. Some manipulations of incentive compatibility constraints allow for a restatement of them as a family of equations. The goal is to embed those into the model. The following result facilitates the simplification:

Lemma 3 (IC) is equivalent to: 1) $\frac{u(r)}{r}-\frac{u(\tilde{r})}{\tilde{r}}=\int_{r}^{\tilde{r}} \frac{Q_{1}(x)}{x^{2}} d x$, for any $[r, \tilde{r}] \in[1 / 2,1]^{2}$; and 2) $Q_{1}(\cdot)$ is a nonincreasing function (or equivalently, $Q_{2}(\cdot)$ is nondecreasing). (IC) also has the following implications: 3) $Q_{1}(\cdot)+Q_{2}(\cdot)$ is nonincreasing on $(1 / 2,1]$ (strictly decreasing when $Q(r)$ varies as $r$ is increased), and that 4$)$ there exists a unique $r^{\prime} \in(1 / 2,1]$ such that $R_{2}(r)>R_{1}(r)$ for all $r \in\left(1 / 2, r^{\prime}\right)$ and $R_{2}(r)<R_{1}(r)$ for all $r>r^{\prime}$.

Proof. See the Appendix.

Point 3) illustrates the trade-off implied by (IC). If an agent wants an additional probability unit of her most-preferred object, she must be willing to sacrifice more than one unit of the non-preferred object. From point 4) in this lemma it can be observed that for low values of $r$ (although higher than 1/2) only the $Q_{1}$-constraints may bind. In cases where $r^{\prime}$ equals 1 , this observation provides a simple way to solve the allocation problem, since the $Q_{2}$-constraints become redundant.

To think about points 1) and 2), consider $u(r) / r=\frac{1-r}{r} Q_{1}(r)+Q_{2}(r)$. (IC) can be divided by $r$ while keeping the inequality unchanged. After such manipulation of $(\mathrm{IC}), u(r) / r$ resembles an interim payoff 
function in auctions, say $\breve{u}(\breve{v}) \breve{Q}(\breve{v})-\breve{T}(\breve{v}),{ }^{15}$ where $\breve{v}$ is the agent's type, $\breve{u}(\breve{v})$ is the utility derived from winning the object for sale, $\breve{Q}(\breve{v})$ is the interim probability of winning the auction, and $\breve{T}(\breve{v})$ is the interim expected payment in numeraire units. Analogously, $Q_{2}(\cdot)$ is being used in our problem as the numeraire that is transferred in exchange of more (or less) $Q_{1}(\cdot)$. Using a standard technique (see the appendix), the numeraire can be solved out as a function of the interim allocation probabilities of the other object.

In that sense, working with $u(r)$ would not have been enough. The standard procedure would yield $u^{\prime}(r)=Q_{2}(r)-Q_{1}(r)$. Consequently, $Q_{2}(r)$ could not have been substituted out in the objective function. In taking $u(r) / r$ instead, I can use point 1$)$ in the lemma above to obtain a relation between any $Q_{2}(r)$, a reference value $u(1) / 1=Q_{2}(1)$ and all $Q_{1}(x)$ 's for $x \in[r, 1]$ :

$$
Q_{2}(r)=Q_{2}(1)-Q_{1}(r) \frac{1-r}{r}+\int_{r}^{1} \frac{Q_{1}(x)}{x^{2}} d x
$$

leading to $u(r)=r Q_{2}(1)+r \int_{r}^{1} \frac{Q_{1}(x)}{x^{2}} d x$.

Solving for $Q_{2}(1)$. The next step is to calculate the value of $u(1) / 1=Q_{2}(1)$.

Definition 1 The distribution function $F$ satisfies the regularity condition (or is regular) if for any $r \in$ $(1 / 2,1], E\left(v_{2} \mid r\right)>\max _{\tilde{r} \in[1 / 2, r]} E\left(v_{1} \mid \tilde{r}\right)$ (equivalently, $E\left(v_{2} \mid r\right)>\max _{r^{\prime} \in[1-r, 1 / 2]} E\left(v_{2} \mid r^{\prime}\right)$, given the symmetry of $F$ ).

Consider two agents $i$ and $j$. Agent $i$ prefers object $h$, while $j$ prefers the other object, $3-h$. Say that the latter agent has less intense preferences than the former (i.e. $\left.\left|r^{j}-1 / 2\right| \leq\left|r^{i}-1 / 2\right|\right)$ ). The regularity condition states that, conditional on their preference intensities, the expected $v_{h}^{i}$ is higher than the expected $v_{h}^{j}$. That is, giving an object to an agent who prefers it provides more welfare than giving that object to an agent who moderately prefers the other object.

The regularity condition is met in quite general scenarios, yet not in all. On the one side, if $E(B \mid r)$ is "sufficiently" decreasing in $r$ on some interval in $(1 / 2,1], F$ would not be regular. ${ }^{16}$ This exception requires that an agent with intense preferences valuates the bundle much less than an agent with moderate preferences. On the other side, it is easy to find intuitive sufficient conditions such that the regularity condition is satisfied. For instance, $F$ meets the regularity condition if $E\left(v_{2} \mid r\right)$ is not decreasing on $(1 / 2,1]$, that is, higher relative preference for the preferred object is corresponded with a weakly higher (conditionally expected) valuation for that object. Examples of widely known distributions that meet this condition are the uniform and the exponential distributions. Other scenarios satisfying the regularity condition are those where if any two agents prefer different objects, conditional on their preference intensities, any agent's expected valuation for her preferred object is higher than the other agent's expected valuation for the same object.

Proposition 1 provides us with a key tool to solve this problem.

\footnotetext{
${ }^{15}$ This notation has no relation with the one I use in the present paper.

${ }^{16}$ In fact, for $r>\tilde{r} \geq 1 / 2$, if $E(B \mid r)<<E(B \mid \tilde{r})$, we could have $E\left(v_{2} \mid r\right)=r E(B \mid r) \leq(1-\tilde{r}) E(B \mid \tilde{r})=$ $E\left(v_{1} \mid \tilde{r}\right)$.
} 
Proposition 1 Under the regularity condition, any solution to the allocation problem meets $R_{1}(1 / 2)=$ $R_{2}(1 / 2)=0$.

Proof. See the Appendix.

The proof starts from a reduced-form mechanism $Q$ that is feasible and incentive compatible while $R_{1}(1 / 2)=R_{2}(1 / 2)>0$. I show that I can construct a feasible, incentive compatible mechanism $\bar{Q}$ such that $\bar{Q}_{2}(r) \geq Q_{2}(r)$ for all $r \in[1 / 2,1]$ (with strict inequality for a positive-measured subset of this domain) and $\bar{Q}_{1}(r) \leq Q_{1}(r)$ only for $r \in[1 / 2, \tilde{r}], \tilde{r} \leq 1$. Moreover, $E\left[\bar{Q}_{1}(\hat{r})+\bar{Q}_{2}(\hat{r})\right] \geq E\left[Q_{1}(\hat{r})+Q_{2}(\hat{r})\right]$. The regularity condition ensures then that $\bar{Q}$ is welfare-improving with respect to $Q$.

One interpretation of this proposition is that any agent that declares to prefer object $h$ over $3-h$ has no chance to obtain object $3-h$ in any optimal mechanism, unless all agents declare to prefer the same object $h$. To illustrate this statement, observe that given that $Q_{2}(\cdot)$ is nondecreasing, a $Q_{2}-$ constraint binding at $r$ implies that for any $\tilde{r}<r, Q_{2}(\tilde{r}) \leq \frac{\Psi(r)^{n}-\Psi(\tilde{r})^{n}}{n[\Psi(r)-\Psi(\tilde{r})]}<\Psi(r)^{n-1} \cdot{ }^{17}$ The last element is the probability that no other agent has a relative preference above $r$. In other words, if some other agent has a relative preference above $r$, an $\tilde{r}$-type agent has no chance to obtain object 2. Applying this argument to the case where $r=1 / 2$ I obtain the statement I aimed to illustrate.

This interpretation of proposition 1 suggests that the optimal mechanism could be understood as a refinement of the ranking mechanism. When some agents prefer object 1 and some other agents prefer object 2, those preferring one object obtain no chance to receive the other good. Ex ante, the planner has to provide agents preferring the same object with the same chances of obtaining that object irrespective of their preference intensities, since the other object cannot be used as numeraire to elicit information on these intensities. Accordingly, the optimal mechanism differs from the ranking mechanism in the way it allocates the objects when all agents prefer the same good (e.g. object 2 if $r \geq 1 / 2$ ).

Using equation (1) and the binding constraint $R_{2}(1 / 2)=0,{ }^{18}$

$$
Q_{2}(1) / 2=\frac{1-(1 / 2)^{n}}{n}+\int_{1 / 2}^{1} Q_{1}(r)\left[\psi(r) \frac{1-r}{r}-\frac{\Psi(r)-1 / 2}{r^{2}}\right] d r
$$

Solving for $Q_{1}(\cdot)$. After having calculated $Q_{2}(1)$, the objective function can be expressed as ${ }^{19}$

$$
O=\kappa+\int_{1 / 2}^{1} \psi(r) Q_{1}(r) C(r) d r
$$

${ }^{17}$ The first inequality arises from $0 \leq R_{2}(\tilde{r})=R_{2}(\tilde{r})-R_{2}(r)=\Psi(r)^{n}-\Psi(\tilde{r})^{n}-n \int_{\tilde{r}}^{r} \psi(x) Q_{2}(x) d x \leq$ $\Psi(r)^{n}-\Psi(\tilde{r})^{n}-n[\Psi(r)-\Psi(\tilde{r})] Q_{2}(\tilde{r})$. The second inequality comes from the fact that $\frac{\Psi(r)^{n}-\Psi(\tilde{r})^{n}}{n[\Psi(r)-\Psi(\tilde{r})]}$ is increasing in $\tilde{r}$ and approaches $\Psi(r)^{n-1}$ as $\tilde{r}$ becomes arbitrarily close to $r$.

${ }^{18}$ To derive (2), we start with $1-(1 / 2)^{n}-n \int_{1 / 2}^{1} \psi(r)\left[Q_{2}(1)-Q_{1}(r) \frac{1-r}{r}+\int_{r}^{1} \frac{Q_{1}(x)}{x^{2}} d x\right] d r=0$.

We then use the formula $\int_{a}^{b} f(x) \int_{x}^{b} g(y) d y d x=\int_{a}^{b} g(y) \int_{a}^{y} f(x) d x d y$, and we finally isolate $Q_{2}(1)$.

${ }^{19}$ Since $O=\int_{1 / 2}^{1} \psi(r) E(B \mid r) u(r) d r$ and $u(r) / r=Q_{2}(1)+\int_{r}^{1} \frac{Q_{1}(x)}{x^{2}} d x$, one obtains $O=$ 
where $\kappa$ denotes a constant, and $C(r)$ is the "criterion function":

$$
\begin{aligned}
C(r) \equiv & \frac{1-r}{r} E\left(v_{2} \mid \hat{r} \geq 1 / 2\right) \\
& -\frac{\Psi(r)-1 / 2}{\psi(r) r^{2}}\left[E\left(v_{2} \mid \hat{r} \geq 1 / 2\right)-E\left(v_{2} \mid r \geq \hat{r} \geq 1 / 2\right)\right]
\end{aligned}
$$

Notice that $C(1 / 2)>C(1)=0$, and that $C(\cdot)$ is continuous all over $[1 / 2,1]$. To understand the criterion function, consider the case in which the expected $v_{2}$ conditional on $r$ is constant. This happens for instance when $F$ is the uniform distribution function. In such a case, object 2 would be a "standard" numeraire good in that it generates the same marginal utility to every agent regardless of her type. The problem to solve would be the one of an efficient auction for object 1, where the criterion function is proportional to $\frac{1-r}{r}$, the marginal rate of substitution $v_{1} / v_{2}$ for an $r$-type agent. And the marginal rate of substitution is the contribution that an agent gives to the objective function in an efficient auction for object 1 .

Unfortunately, the expected $v_{2}$ conditional on $r$ is typically not constant. The criterion function becomes more complex because the planner (who allocates both objects) cannot ignore that the numeraire good generates a utility that depends on the agent's type $r$. Let us denote her marginal rate of substitution as $m=\frac{1-r}{r} \in[0,1]$, with distribution function $G(m)$ and associated density $g(m) . \frac{\Psi(r)-1 / 2}{\psi(r) r^{2}}$ is the inverse hazard rate of the marginal rate of substitution $\frac{1-G(m)}{g(m)}$. In the literature on auctions, the "virtual valuation" is a concept that identifies the contribution a bidder makes to the seller's expected revenues. The "virtual marginal rate of substitution" associated with $m$ is equal to $m-\frac{1-G(m)}{g(m)}$. The criterion function divided by $E\left(v_{2} \mid \hat{r} \geq 1 / 2\right)$ lies always above the marginal rate of substitution. Yet no other general statement about the shape of the criterion function can be made.

Since $R_{2}(1 / 2)=0$, the $Q_{1}$-constraints simplify to:

$$
R_{1}(r)=(1 / 2)^{n}-[1-\Psi(r)]^{n}-n \int_{1 / 2}^{r} \psi(x) Q_{1}(x) d x \geq 0, r \in[1 / 2,1]
$$

$$
\begin{aligned}
& \hline \int_{1 / 2}^{1} \psi(r) E\left(v_{2} \mid r\right)\left[Q_{2}(1)+\int_{r}^{1} \frac{Q_{1}(x)}{x^{2}} d x\right] d r \text {. After having used equation }(2), \text { it yields } \\
& \qquad=\kappa+\int_{1 / 2}^{1} 2 \psi(r) E\left(v_{2} \mid r\right) d r \\
& \cdot \int_{1 / 2}^{1} Q_{1}(r)\left[\psi(r) \frac{1-r}{r}-\frac{\Psi(r)-1 / 2}{r^{2}}\right] d r \\
& +\int_{1 / 2}^{1} \psi(r) E\left(v_{2} \mid r\right) \int_{r}^{1} \frac{Q_{1}(x)}{x^{2}} d x d r
\end{aligned}
$$

where $\kappa$ is a constant. Using the formula in the previous footnote, the objective function becomes a constant plus the integral of $Q_{1}(r) \psi(r) C(r)$, where $C(r)$ contains the remaining factors of the integrand. 
The $Q_{2}$-constraints need not be rewritten here for the purposes of the solution. The problem of maximizing (3) subject to (4) and to nonincreasing $Q_{1}(\cdot)$ is analogous to the reduced-form version of an efficient auction problem. The planner decides how to allocate object 1 among types in $[1 / 2,1]$, under the constraint that the presence of an agent whose type belongs to $[0,1 / 2)$ makes it impossible for types in $[1 / 2,1]$ to obtain that object. ${ }^{20}$ In solving this problem, the standard Myerson (1981) "ironing" technique is applied over $C(\cdot) .{ }^{21}$ The final result of this subsection states a sufficient condition allowing us to safely ignore the $Q_{2}$-constraints.

Proposition 2 Assume that any optimal mechanism satisfies $R_{2}(1 / 2)=0$. Let $F$ be such that $E(C(\hat{r})$ $\mid \hat{r} \geq r) \geq 0$ for any $r \geq 1 / 2$. Then, in order to solve for the function $Q_{1}$, the planner has to maximize $O$ in equation (3) subject to (4) and to $Q_{1}(\cdot)$ being nonincreasing, ignoring the $Q_{2}$-constraints. In order to solve for the function $Q_{2}$, the planner has to use the already solved $Q_{1}^{*}$, jointly with equations (1) and (2).

Proof. Given $E(C(\hat{r}) \mid \hat{r} \geq r) \geq 0$ for any $r \geq 1 / 2$, the solution to the suggested problem yields $R_{1}(1)=0$ (that is, all objects are finally given). Since $R_{2}(1)=0$ by construction, we have $R_{2}(1)-R_{1}(1)=0$. By construction again, $R_{2}(1 / 2)-R_{1}(1 / 2)=0$. By this and point 4 in lemma $2, R_{2}(\cdot)-R_{1}(\cdot)$ cannot be negative in the whole domain $[1 / 2,1]$. Since $R_{1}(\cdot)$ is nonnegative in that domain, $R_{2}(\cdot)$ cannot be negative, and therefore the $Q_{2}-$ constraints are met.

The sign of $E(C(\hat{r}) \mid \hat{r} \geq r)$ determines whether it is optimal to give object 1 away if all agents' types are inside $[r, 1]$. If that sign is negative, the planner may not give that object away, and this possibility may make proposition 2 fail. If the planner cannot commit to a mechanism that may "burn" objects, or if she is just constrained to give all the objects away, then $R_{1}(1)=0$ (the last feasibility constraint binds), and the proposition holds regardless the sign of the conditionally expected $C(\cdot)$.

Lastly, I assume that the criterion function is nonincreasing. This is analogous to the usual assumption that the virtual valuation is nondecreasing in auction theory. Since $C(1)=0$, if $C(r)$ is nonincreasing in $r$ for all $r \in[1 / 2,1]$, then $E(C(\hat{r}) \mid \hat{r} \geq r)$ is positive for every $r \geq 1 / 2$. The following corollary holds.

Corollary 1 Assume that any optimal mechanism satisfies $R_{2}(1 / 2)=0$. Let $C(r)$ be nonincreasing in every $r \in[1 / 2,1]$. Then there exists an optimal mechanism $q^{*}$ such that $R_{1}(r)=0$ for any $r \in[1 / 2,1]$. Thus, $Q_{1}^{*}(r)=[1-\Psi(r)]^{n-1}, \forall r \in[1 / 2,1]$.

${ }^{20}$ In ex-post probabilities, the planner maximizes $\int_{1 / 2}^{1} \ldots \int_{1 / 2}^{1} \sum_{i=1}^{n} q_{1}^{i}\left(r^{1}, \ldots, r^{n}\right) C\left(r^{i}\right) \prod_{i=1}^{n} d \Psi\left(r^{i}\right)$ subject to $\sum_{i=1}^{n} q_{1}^{i}\left(r^{1}, \ldots, r^{n}\right) \leq 1$ for any $\left(r^{1}, \ldots, r^{n}\right) \in[1 / 2,1]^{n}$ and to $Q_{1}(\cdot)$ being nonincreasing.

${ }^{21}$ Define $K(1-\pi) \equiv C\left[\Psi^{-1}(\pi)\right] . K(\cdot)$ has $[0,1 / 2]$ as its domain. Take $\tilde{K}(p)$ as the derivative of the convex hull of the integral of $K(\cdot)$ from 0 to $p$. We obtain the ironed criterion function as $\tilde{C}(r)=\tilde{K}(1-\Psi(r))$. We optimally assign object 1 to the agent with the highest (positive) value of the ironed criterion function, breaking ties evenly. 
The corollary can be understood as follows: if all agents prefer the same good and the criterion function is nonincreasing, the optimal mechanism assigns the non-preferred good to the agent with more moderate preferences. In the examples below, both the uniform and the exponential valuation distributions meet this condition on $C(\cdot)$. This corollary is useful when comparing the optimal mechanism to the ranking mechanism.

\section{Implementation and comparison with respect to the Ranking Mechanism}

In what follows, I assume that the criterion function is nonincreasing. The purpose of this subsection is to find mechanisms (that is, ex-post probability functions $q(\cdot)$ ) that implement the interim probabilities of the optimal mechanism. To start with, I treat the case in which there are two agents. Below I define two mechanisms that achieve the desired results.

Definition 2 The auction mechanism (with two agents). Each agent $i$ reports her preferred object $h^{i} \in\{1,2\}$ and a bid $b^{i} \in[0,1]$ to the planner in a sealed envelope. If the agents report to prefer different objects, each agent obtains her reportedly preferred object. If both agents reportedly prefer the same object $h$, then: 1) an endowment probability 1/2 of obtaining that object is given to each agent, and 2) the other object $(3-h)$ is auctioned off and given to the agent with the highest bid, who pays her bid (up to 1/2) in probability units of obtaining object $h$, and this payment is transferred to the agent who loses the auction (who does not pay her bid).

Definition 3 The market mechanism (with two agents). For each object $h \in\{1,2\}$, each agent is given an equal endowment probability 1/2 of obtaining that object. Each agent $i$ announces a price for object 2, $p^{i} \in[0,1]$. Announcements are simultaneous. If $p^{i}<1 / 2 \leq p^{-i}$, the planner sets equilibrium prices $p_{1}^{*}$ and $p_{2}^{*}$ for objects 1 and 2 respectively, such that $p_{1}^{*}=p_{2}^{*}=1 / 2$. Agent $i$ sells her endowment (in probability units) of object 2 and buys agent - $i$ 's endowment of object 1. Agent $-i$ buys and sells oppositely so as to clear the market. If $1 / 2 \leq p^{i}<p^{-i}$, the planner sets equilibrium prices $p_{2}^{*}=p^{i}$ and $p_{1}^{*}=1-p^{i}$. Agent $i$ sells $\frac{1-p^{i}}{2 p^{i}}$ from her endowment (in probability units) of object 2 and buys agent $-i$ 's endowment of object 1. Agent $-i$ buys and sells so as to clear the market. The procedure is symmetric when both announced prices lie below 1/2: given $1 / 2>p^{i}>p^{-i}$, the planner sets equilibrium prices $p_{2}^{*}=p^{i}$ and $p_{1}^{*}=1-p^{i}$, and agent $i$ sells $\frac{p^{i}}{2\left(1-p^{i}\right)}$ from her endowment (in probability units) of object 1 and buys agent $-i$ 's endowment of object 2. Finally, if $p^{i}=p^{-i}$, the equilibrium prices are set as $p_{2}^{*}=p^{i}$ and $p_{1}^{*}=1-p^{i}$, and agents do not trade.

It is easy to check that both mechanisms have equilibria yielding identical outcomes. In the Appendix, I derive the symmetric equilibrium bid function $B(\cdot)$ in the auction. In the market mechanism, agents do not behave competitively, that is, announced prices $P(\cdot)$ may not reflect real preference parameters $(P(r) \neq r)$. I 
explain in the Appendix that in a symmetric equilibrium, the price announcement function $P(\cdot)$ for $r>1 / 2$ follows the formula $\frac{1-P(r)}{P(r)}=E_{N}\left(\max _{i \in\{1,2\}} \frac{1-\hat{r}^{i}}{\hat{r}^{i}} \mid \frac{1-r}{r}>\max _{i \in\{1,2\}} \frac{1-\hat{r}^{i}}{\hat{r}^{i}}\right)$. The symmetric equilibrium bid function $B(\cdot)$ in the auction game is equivalent to $\frac{1-P(\cdot)}{2 P(\cdot)}$ (observe that the equilibrium bid does not exceed $\left.1 / 2\right) .{ }^{22}$ Observe that when both agents prefer the same object, both equilibria imply that the non-preferred object is received by the agent with more moderate preferences. Efficiency is reached in that sense even though agents behave strategically.

Now, let us say that a mechanism (symmetric Bayesian Nash) implements the optimal mechanism if its induced game has a symmetric Bayesian Nash equilibrium in which, after being played, each agent's interim expected allocation equals the interim allocation probabilities of the optimal mechanism.

Proposition 3 Let $n=2$ with regular $F$ and nonincreasing criterion function. Then both the auction mechanism and the market mechanism (symmetric Bayesian Nash) implement the optimal mechanism.

Proof. See the Appendix.

The optimal mechanism differs from the Ranking Mechanism when both agents prefer the same object. Two examples illustrate the welfare gains that can be achieved when the ranking mechanism is replaced by the auction (or the market) mechanism. In the first example shown here, the marginal distribution of the valuation for each object is uniform. In the second example, each object valuation is drawn from the same exponential distribution. Both examples in this subsection satisfy the requirements of propositions 1 and 2 (and its corollary).

Example 1 The uniform distribution: $\tilde{F}(v)=v, v \in[0,1]$. For $r \geq 1 / 2$, the density of $r$ is $\psi(r)=\frac{1}{2 r^{2}}$. Normalize the per agent ex-ante expected utility in the first best (perfect information) case to 1. By even randomization, the no-information case, each agent obtains an ex-ante expected payoff that is normalized to 0 . Under this normalization, the optimal mechanism yields a per agent ex-ante expected payoff of $2 / 3$. The ranking mechanism obtains $1 / 2$.

Example 2 The exponential distribution: $\tilde{F}(v)=1-e^{-\lambda v}, v \in[0, \infty), \lambda>0$. In this case, $r$ is distributed as a uniform $U[0,1]$, with density $\psi(r)=1$. Under the normalization of Example 1, the optimal mechanism gives a welfare of $2(1-\log 2) \simeq .6137$. The ranking mechanism yields $1 / 2$ instead.

Next, I consider the cases where $n>2$, in which implementation is more complex. Consider for instance a generalization of the auction mechanism depicted above. If not all the agents declare to prefer the same object, each object is randomly assigned to an agent who reports preferring it. If all agents declare to prefer the same object $h$, then: 1) an endowment probability $1 / n$ of obtaining that object is given to each agent,

${ }^{22}$ When $r<1 / 2$, then $\frac{P(r)}{1-P(r)}=E_{N}\left(\max _{i \in\{1,2\}} \frac{\tilde{r}^{i}}{1-\tilde{r}^{i}} \mid r>\tilde{r}^{1}, r>\tilde{r}^{2}\right)$, and $B(r)=\frac{P(r)}{2[1-P(r)]}$. 
and 2) the other object $3-h$ is auctioned off and given to the agent with the highest (sealed) bid, who pays her bid in probability units of obtaining object $h$, and this payment is evenly transferred to the agents who lose the auction (they do not pay their bids).

Ignoring the endowment constraint, the symmetric equilibrium of this first-price auction gives a bid function $B(r)=\frac{n-1}{n} E_{N}\left(\max _{i \in N} \frac{1-\hat{r}^{i}}{\hat{r}^{i}} \mid \frac{1-r}{r}>\max _{i \in N} \frac{1-\hat{r}^{i}}{\hat{r}^{i}}\right)$ (when everyone prefers object 2). ${ }^{23}$ However, when $n>2$, the unconstrained equilibrium bid may exceed the endowment probability $1 / n .{ }^{24}$ This is intuitive since the equilibrium bids become more aggressive when each agent faces more opponents, while the budget limit shrinks as the number of agents increases. With this constraint, the equilibrium bid function is capped by the endowment, and hence the auction may not yield an efficient allocation of the non-preferred object. A similar problem occurs with a generalization of the market mechanism.

In a sense, this feasibility problem occurs due to insufficiently developed insurance markets. An agent could be interested in buying a security that gives her extra endowment in states of the world where an auction is to be played, in exchange of lower endowments in other states of the world. This way she could optimally bid ignoring the endowment constraints, leading to an efficient assignment of the non-preferred object. The mechanism I present here solves this insufficiency problem. ${ }^{25}$

Definition 4 The insured auction mechanism (for more than two agents) . Each agent $i$ reports her preferred object $h^{i} \in\{1,2\}$ and a bid $b^{i} \in[0,1]$ to the planner in a sealed envelope. Let $B(r)=$ $\frac{n-1}{n} E_{N}\left(\max _{i \in N} \frac{1-\hat{r}^{i}}{\hat{r}^{i}} \mid \frac{1-r}{r}>\max _{i \in N} \frac{1-\hat{r}^{i}}{\hat{r}^{i}}\right)$ for $r \geq 1 / 2$ and $B(r)=\frac{n-1}{n} \cdot E_{N}\left(\max _{i \in N} \frac{\hat{r}^{i}}{1-\hat{r}^{i}} \mid \frac{r}{1-r}>\max _{i \in N} \frac{\hat{r}^{i}}{1-\hat{r}^{i}}\right)$ for $r<1 / 2$. For any bid $b$, let $V(b)=\max \{0, b-1 / n\}$. The allocation proceeds as follows:

1) If all agents declare to prefer the same object, each agent obtains an initial endowment (in probability units) of $1 / n$ of that object, and an auction gives the non-preferred object to the agent with the highest bid. The winner $i$ pays $b^{i}-V\left(b^{i}\right)$ in probability units of the most-preferred object, which is evenly split among the losers of the auction (who do not pay their bids).

2) If all but one (call her $j$ ) agents declare to prefer the same object $h$, an initial endowment $\frac{1}{n-1}$ of that object is given to these agents. Then the agent $i$ with the highest bid among them pays $\frac{1-\Psi\left(B^{-1}\left(b^{i}\right)\right)}{(n-1) / 2} \cdot V\left(b^{i}\right)$ in probability units of that object $h$, and this payment is evenly split among the other agents who claimed to prefer that object. The remaining agent $j$ obtains the remaining object $3-h$.

3) If neither the conditions in 1) nor 2) are met, each object is evenly split (in probability units) among those agents who declare to prefer it.

${ }^{23}$ This result can be found for instance in McAfee and McMillan (1992). I also derive it in the Appendix. ${ }^{24}$ Any efficient auction format would yield the same problem as long as $\frac{n-1}{n} E_{N}\left(\max _{i \in\{1, \ldots, n\}} \frac{1-\hat{r}^{i}}{\hat{r}^{i}}\right)>\frac{1}{n}$.

${ }^{25}$ This is one possible solution. Alternatively, the market mechanism could be appropriately extended to include securities that led markets to the desired outcome. The approach I take in the paper is useful in that it obtains a mechanism that can be easily compared to the Ranking Mechanism. 
The function $V\left(b^{i}\right)$ characterizes a security (or a voucher) an agent cashes in states of the world in which an auction is run and she becomes the winner. The price for that security is characterized in point 2) of the previous definition: there are states of the word in which this agent sacrifices part of its endowment with no compensation. To calculate this price the planner needs to know the equilibrium bid function in an auction with unconstrained budgets. Notwithstanding this, the aforementioned mechanism is feasible ${ }^{26}$ and achieves incentive efficient allocations. It is shown in the Appendix that this insurance policy has zero effect on any agent's ex-ante allocated probabilities while allowing for efficient auctions.

Proposition 4 For $n>2$ with regular $F$ and a nonincreasing criterion function, the insured auction mechanism (symmetric Bayesian Nash) implements the optimal mechanism.

Proof. See the Appendix.

Apart from the insurance policy depicted above, this optimal mechanism differs from the Ranking Mechanism only in the two cases where all agents prefer the same object. This happens with probability $\frac{1}{2^{n-1}}$. Thus the welfare difference between the optimal mechanism and the ranking mechanism tends to vanish when the number of agents is large.

Corollary 2 In a regular environment with a nonincreasing criterion function, the welfare difference between the optimal mechanism and the ranking mechanism is of the order of $\frac{1}{2^{n-1}}$ and collapses to 0 as $n \rightarrow \infty$.

With many agents, one could simply use the ranking mechanism without being afraid of big efficiency losses. With few agents, the use of the optimal mechanism is clearly justified. In the two-agent case, the optimal mechanism is implemented via intuitive and rather simple mechanisms that do not require the planner to know the distribution of preferences.

\section{The two-school random assignment problem}

One of the features of the problem so far analyzed is that it poses no restrictions on the number of objects that will be allocated to each agent. In particular, it may be the case that both objects are allocated to the same agent, or that some object is not allocated to any agent. While this is fine in many settings, such as collusion mechanisms, in some cases this assumption is inappropriate. A paradigmatic case, from which this Section takes its name, consists of the assignment of children to public schools. Naturally, no child can be allocated to more than one school, and all children must be assigned.

The two-school random assignment problem is introduced as follows. There is a number $n$ of children, and each of them is to be assigned to exactly one of two schools. School 1 has $n_{1} \in\{1, \ldots, n-1\}$ slots to be

${ }^{26}$ Since the maximum bid is 1 , the maximum payment in point 2 ) of the definition is $1 / n$, while the initial endowment in that case is $\frac{1}{n-1}$. 
fulfilled, and school 2 has $n_{2}=n-n_{1}$ vacancies. It is assumed that schools' preferences will not play a role here, so this is a one-sided matching problem. Child $i$ parents' preferences over schools are represented by valuations $v_{1}^{i}$ and $v_{2}^{i}$, as in the original game, and these are private information. Valuations are i.i.d. among parents, and marginal distributions are identical for the two schools. Parents are indifferent among different slots in the same school.

The mechanism designer asks for information about parents' preferences, and allocates assignment probabilities $q_{h}^{i}(\cdot), \forall i \in\{1, \ldots, n\}, \forall h \in\{1,2\}$, as a function of revealed information. $q_{h}^{i}$ is the probability that children $i$ is allocated a slot in school $h$. The preliminary results of Section 2 still apply, so without loss of generality $q_{h}^{i}$ will depend only on the vector $\left(r^{1}, \ldots, r^{n}\right)$, where $r^{i}$ is defined as in Section 2. $Q_{h}^{i}\left(r^{i}\right)$, the reduced-form probability, is also defined as in Section 2, for any $i$ and $h$.

The constraint that each child is assigned to exactly one school implies that for any $\left(r^{1}, \ldots, r^{n}\right) \in[0,1]^{n}$ the matrix $q\left(r^{1}, \ldots, r^{n}\right) \equiv\left[q_{h}^{i}\left(r^{1}, \ldots, r^{n}\right)\right]_{i, h}$ satisfies $\sum_{h} q_{h}^{i}\left(r^{1}, \ldots, r^{n}\right)=1$ for any $i$. A consequence of this property of the random assignment is that for any $i$ and $r^{i}$, it must hold that $Q_{2}^{i}\left(r^{i}\right)=1-Q_{1}^{i}\left(r^{i}\right)$, a constraint that was not present in the previous sections. Child $i$ parents' interim utility is therefore $U^{i}\left(r^{i}, B^{i}\right) \equiv$ $B^{i}\left[Q_{1}^{i}\left(r^{i}\right)\left(1-r^{i}\right)+Q_{2}^{i}\left(r^{i}\right) r^{i}\right]=B^{i}\left[r^{i}+Q_{1}^{i}\left(r^{i}\right)\left(1-2 r^{i}\right)\right]$. Since it only depends on one reduced form probability, the next result follows immediately.

Proposition 5 In the two-school random assignment problem, BIC is equivalent to the following properties for any $i \in\{1, \ldots, n\}$ and any pair $\left.(r, \tilde{r}) \in[0,1]^{2}, r \geq \tilde{r}: 1\right)$ if $\operatorname{sign}(r-1 / 2)=\operatorname{sign}(\tilde{r}-1 / 2)$, then $Q^{i}(r)=Q^{i}(\tilde{r})$, and 2) if $r \geq 1 / 2 \geq \tilde{r}$, then $Q_{1}^{i}(r) \leq Q_{1}^{i}(\tilde{r})$ (and therefore $Q_{2}^{i}(r) \geq Q_{2}^{i}(\tilde{r})$ ).

Proof. After taking $Q_{2}^{i}\left(r^{i}\right)=1-Q_{1}^{i}\left(r^{i}\right)$ into account, the BIC constraints can be restated as: for any $i \in\{1, \ldots, n\}$ and any pair $(r, \tilde{r}) \in[0,1]^{2}$, where without loss of generality $r \geq \tilde{r}$, we have $Q_{1}^{i}(r)(1-2 r) \geq$ $Q_{1}^{i}(\tilde{r})(1-2 r)$ and $Q_{1}^{i}(r)(1-2 \tilde{r}) \leq Q_{1}^{i}(\tilde{r})(1-2 \tilde{r})$. It is easy to check that this is equivalent to the stated properties.

The main consequence is apparent. In the problem under analysis, if a mechanism is Bayesian incentive compatible, then it can only elicit useful information on the agents' ordinal preferences.

Corollary 3 The Ranking Mechanism (in a version that ensures exactly one slot per child) solves the twoschool random assignment problem in environments characterized by Private Values and identical marginals.

The way the ranking mechanism is implemented here is as follows. Let the number of parents ${ }^{27}$ claiming to prefer school 1 be denoted by $n_{1}^{*}$. If $n_{1}^{*}=n_{1}$, then there is a perfect match between preferences and slots. If $n_{1}^{*}>n_{1}$, then all children whose parents claim to prefer school 2 are ensured a placement in that school. The remaining slots are allocated among the unassigned children by even randomization. If $n_{1}^{*}<n_{1}$, then the ones that prefer school 1 are the ones that are ensured a place in that school.

${ }^{27}$ Each child's parents count as one individual. 
The corollary stems from this fact: allocating each slot at a given school to an agent that prefers that school whenever possible generates more welfare than allocating otherwise. And this is precisely what this version of the ranking mechanism does. With two schools, several school assignment mechanisms that are studied in the school choice literature (Deferred Acceptance, the Boston Mechanism, the Pseudomarket) yield the same random allocation as the ranking mechanism does if the schools have no priorities across the students.

\section{Extensions}

The results obtained through the present paper could be extended at least in two dimensions, namely a higher number of objects and ex-ante non-identical objects. A brief discussion on what could happen when we relax the assumptions I take in this paper is provided in this Section, in the understanding that a more general solution is still pending. I simply suggest ideas that give some clues for future research.

When the number of objects is increased, the complexity burden of the problem at hand becomes hardly manageable. As previously mentioned, the literature on auctions has not yet made definite progress in the design of revenue-maximizing multi-object auctions. Likewise, in a non-transfer allocation problem with more than two objects, even if the numeraire good is correctly chosen, it is not clear how this numeraire is optimally used to purchase probability units of the other goods. It is interesting though to explore suboptimal but simple mechanisms that improve over mechanisms that simply use information on the agents' ordinal preferences. In that sense, the Boston Mechanism obtains an interesting improvement over Deferred Acceptance (a mechanism that elicits information on ordinal preferences only) in school choice problems (Abdulkadiroğlu, Che and Yasuda, 2009). Policymakers' options are being enriched by these recent contributions.

Likewise one could consider allocating objects that may not be ex-ante identical. It should be stressed that the assumption of ex-ante identical objects highlights the role of information transmission and trade-off rules in allocation mechanisms. Consider for instance the extreme opposite case in which one of the objects (the popular object) is always preferred to the other. Assuming other conditions (regularity, monotone criterion function), optimally the planner should split the popular object into equal allocation probabilities and auction the "unpopular" object off using the popular object as numeraire. This auction is efficient with two agents as we have seen in previous sections. However, given the budget constraint, efficiency is not guaranteed when the number of agents exceeds two. The planner's ability to avoid the budgetary problems is severely limited in this setup. When objects are ex-ante identical, the planner could impose an insurance scheme based on states of the world where not all agents prefer the same object. This option is not available when one of the objects is always preferred by everyone. Consequently, the efficient allocation of the non-preferred object may not be feasible. 
Other assumptions such as Independent Private Valuations (although valuations were allowed to be correlated between objects) and no synergies have been made here. The effects of relaxing these assumptions are still unknown. The introduction of synergies substantially complicates the allocation problem. If the synergy value is proportional to the previously defined bundle valuation, all the relevant information used in the optimal mechanism would still consist of relative preference parameters. No more results could be stated at this moment. Further research will address all these additional matters.

\section{Conclusions}

Economists have extensively analyzed the problem of allocating several indivisible objects to several agents, under the constraint that no monetary transfers can be made. The ranking mechanism, where the allocation of each object is solely a function of the agents' ordinal preferences, has been suggested as a satisfactory solution, due to its simplicity and efficiency properties.

Mechanisms eliciting information on cardinal preferences are nevertheless preferable. When Bayesian (or interim) incentive compatible mechanisms are analyzed, obtaining information on agents' marginal rates of substitution between objects becomes valuable in determining the allocation decision. Bayesian cardinal mechanisms obtain better random allocations than ordinal mechanisms. The improvement is relatively more important the smaller is the number of objects to be allocated and the smaller is the number of agents that participate.

Solving for the ex-ante utilitarian-optimal cardinal mechanism (the optimal mechanism in this paper) is a cumbersome task due to the multidimensionality of types. This paper has analyzed the two-object, $n$-symmetric-agent, Independent-Private-Valuation case, and has provided a solution method. The main idea of the solution is rather simple: one of the objects can be used as numeraire (in probability units) in order to purchase probability units of the other object. Implementation has been characterized for a wide set of environments. With two agents, both a market mechanism and an auction mechanism suffice. With more agents, incomplete insurance markets deter the previous mechanisms from attaining efficient allocations in all cases. The agent who should obtain one of the objects under the optimal mechanism may not have enough of the numeraire good to purchase the former object. The planner must complete the auction mechanism with an insurance policy that guarantees feasibility of the ex-ante efficient allocation.

I construct examples in order to compare the optimal mechanism to the ranking mechanism in terms of welfare. Let us normalize the welfare obtained under even randomization of both objects to 0 , and the welfare obtained by a perfectly informed planner to 1 . Under this normalization, with two agents and uniformly distributed valuations, the ranking mechanism obtains a welfare of $1 / 2$ and the optimal mechanism $2 / 3$. The examples show that with few agents the optimal mechanism significantly differs from the ranking mechanism. As the number of agents grow large, the difference between the optimal mechanism and the 
ranking mechanism diminishes and finally vanishes in the limit.

Interestingly, when the problem is additionally constrained to ensure one (and only one) object per agent, incentive compatibility precludes any use of cardinal information, in the two-object case. The ranking mechanism turns out to be an optimal mechanism.

There is further interesting work to be done in this and related topics. The optimal mechanism problem shall be extended to the allocation of more than two objects, and to the allocation of ex-ante non-identical objects, being one of them more popular than the other. It is also interesting to further explore mechanisms in which the planner does not need information about the priors.

\section{References}

[1] Abdulkadiroğlu A., Che Y. and Yasuda Y. (2009) "Resolving Conflicting Preferences in School Choice: The'Boston'Mechanism Reconsidered", American Economic Review, forthcoming.

[2] Abdulkadiroğlu A. and Loertscher S. (2007) Dynamic House Allocations, mimeo, Duke University and University of Melbourne.

[3] Armstrong M. (2000) "Optimal multi-object auctions", The Review of Economic Studies 67, 455-481.

[4] Bogomolnaia A. and Moulin H. (2001) "A New Solution to the Random Assignment Problem", Journal of Economic Theory 100, 295-328.

[5] Border K.C. (1991) "Implementation of reduced form auctions: a geometric approach", Econometrica $59,1175-1187$.

[6] Börgers T. and Postl P. (2006) Efficient Compromising, mimeo, University of Michigan and University of Birmingham.

[7] Campbell C.M. (1998) "Coordination in auctions with entry", Journal of Economic Theory 82, 425-450.

[8] Casella A. (2005) "Storable votes", Games and Economic Behavior 51, 391-419.

[9] Casella A. and Gelman A. (2005) A Simple Scheme to Improve the Efficiency of Referenda, mimeo, Columbia University.

[10] Clarke E. (1971) "Multipart pricing of public goods", Public Choice 8, 19-33.

[11] Condorelli D. (2010) "What money can't buy: optimal allocations with priority lists, lotteries and queues", International Economic Review, forthcoming. 
[12] Ehlers L. and Klaus B. (2003) "Coalitional strategy-proof and resource-monotonic solutions for multiple assignment problems", Social Choice and Welfare 21, 265-280.

[13] Gibbard A. (1973) "Manipulation of voting schemes: a general result", Econometrica 41, 587-602.

[14] Groves T. (1973) "Incentives in teams", Econometrica 41, 617-631.

[15] Harsanyi J.C. (1955) "Cardinal welfare, individualistic ethics, and interpersonal comparisons of utility", The Journal of Political Economy 63, 309-321.

[16] Hortalà-Vallvé R. (2007) Inefficiencies on Linking Decisions, mimeo, Oxford University and STICERD.

[17] Hylland A. and Zeckhauser R. (1979) "The efficient allocation of individuals to positions", Journal of Political Economy 87, 293-313.

[18] Krishna V. and Rosenthal R.W. (1996) "Simultaneous auctions with synergies", Games and Economic Behaviour 17, 1-31.

[19] Kwasnica A. (2002) A Theory of Collusion in Multiple Object Simultaneous Auctions, mimeo, Penn State University.

[20] Levin J. (1997) "An optimal auction for complements", Games and Economic Behavior 18, 176-192.

[21] Mas-Colell A., Whinston M.D. and Green J.R. (1995), Microeconomic Theory, Oxford: Oxford Press.

[22] Maskin E.S. (2000) "Auctions, development and privatization: Efficient auctions with liquidityconstrained buyers", European Economic Review 44, 667-681.

[23] McAfee P. and McMillan J. (1992) "Bidding Rings", American Economic Review 82, 579-599.

[24] McLean R. and Postlewaite A. (2006) Implementation with Interdependent Valuations, University of Pennsylvania, PIER Working Paper Archive 06-007.

[25] Menezes F.M. and Monteiro P.K. (2003) "Synergies and price trends in sequential auctions", Review of Economic Design 8, 85-98.

[26] Myerson R.B. (1981) "Optimal auction design", Mathematics of Operation Research 6, 58-72.

[27] Olson M. (1991) Dominant and Nash Strategy Mechanisms for the Assignment Problem, Social Science Working Paper $n^{o} 770$, California Institute of Technology.

[28] Pápai S. (2000) "Strategyproof multiple assignment using quotas", Review of Economic Design 5, 91$105-$ 
[29] Pápai S. (2001) "Strategyproof and nonbossy multiple assignments", Journal of Public Economic Theory $3,257-271$.

[30] Pesendorfer M. (2000) "A study of collusion in first-price auctions", Review of Economic Studies 67, $381-411$.

[31] Saari, D. (2000) "Mathematical structure of voting paradoxes", Economic Theory 15, 1-102.

[32] Satterthwaite M.A. (1975) "Strategy-proofness and Arrow's conditions: existence and correspondence theorems for voting procedures and social welfare functions", Journal of Economic Theory 10, 187-217.

[33] Vickrey W. (1961) "Counterspeculation, auctions and competitive sealed tenders", Journal of Finance $16,8-37$.

\section{Appendix}

\section{Proof. Lemma 2.}

1) and 2): divide (IC) by $r$ (notice that the inequalities still hold) and proceed to a standard manipulation of these new constraints, following for instance the technique in Mas-Colell, Whinston and Green $(1995,888-$ 889, proof of proposition 23.D.2).

3): Consider $\tilde{r}>r>1 / 2$. Dividing (IC) by $r$, we have $Q_{2}(\tilde{r})-Q_{2}(r) \leq \frac{1-r}{r}\left[Q_{1}(r)-Q_{1}(\tilde{r})\right]$. Adding $Q_{1}(\tilde{r})-Q_{1}(r)$ to both sides, I obtain $\left[Q_{1}(\tilde{r})+Q_{2}(\tilde{r})\right]-\left[Q_{1}(r)+Q_{2}(r)\right] \leq \frac{1-2 r}{r}\left[Q_{1}(r)-Q_{1}(\tilde{r})\right] \leq 0$, with the last inequality being strict when $Q_{1}(r) \neq Q_{1}(\tilde{r})$ because of point 2$)$ in this lemma.

4): Define, for any object $h$ and for any $p \in[1 / 2,1], \hat{R}_{h}(p) \equiv R_{h}\left[\Psi^{-1}(p)\right]$, and $\hat{Q}_{h}(p) \equiv Q_{h}\left[\Psi^{-1}(p)\right]$. Given 3), it is easy to see that $\hat{R}_{2}(p)-\hat{R}_{1}(p)=(1-p)^{n}-p^{n}+n \int_{1 / 2}^{p}\left[\hat{Q}_{1}(\pi)+\hat{Q}_{2}(\pi)\right] d \pi$ is strictly concave on $[1 / 2,1]$. Thus $R_{2}(\cdot)-R_{1}(\cdot)$ is first strictly increasing up to some point $\tilde{r} \in(1 / 2,1]$ and then strictly decreasing for values $r>\tilde{r}$. We know $R_{2}(1 / 2)=R_{1}(1 / 2)$ and $R_{2}(1)=0 \leq R_{1}(1)$ by construction of these functions. Thus $R_{2}(\cdot)-R_{1}(\cdot)$ must have a unique point $r^{\prime} \in(\tilde{r}, 1]$ such that $R_{2}(r)-R_{1}(r)>0$ if $1 / 2<r<r^{\prime}$ and $R_{2}(r)-R_{1}(r)<0$ if $r>r^{\prime}$.

\section{Proof. Proposition 1.}

Fix a feasible, Bayesian incentive compatible (BIC) reduced-form (direct) mechanism $Q$ that satisfies $R_{1}(1 / 2)=R_{2}(1 / 2)>0$. The proof consists of the construction of an alternative feasible, BIC reduced-form (direct) mechanism $\bar{Q}$ that obtains a higher ex-ante expected welfare than $Q$. As we will see, the new reducedform allocation $\bar{Q}$ is welfare-improving with respect to $Q$ because $\bar{Q}_{2}(\cdot) \geq Q_{2}(\cdot)$ (with strict inequality for a positive-measured subset of the domain) while $\int_{1 / 2}^{1} \psi(x)\left[\bar{Q}_{1}(x)+\bar{Q}_{2}(x)\right] d x \geq \int_{1 / 2}^{1} \psi(x)\left[Q_{1}(x)+Q_{2}(x)\right] d x$. 
Given the regularity condition, the welfare gain induced by raising $Q_{2}$ is higher than the welfare loss induced by the reduction of $Q_{1}$.

Along the proof I use the following notation. Associated with $Q$ there are constraint functions $R_{1}$ and $R_{2}$ and a utility function $u(r) \equiv r Q_{2}(r)+(1-r) Q_{1}(r)$. Associated with $\bar{Q}$ there are $\bar{R}_{1}, \bar{R}_{2}$ and $\bar{u}(r) \equiv r \bar{Q}_{2}(r)+(1-r) \bar{Q}_{1}(r)$. For any function $g(\cdot)$, I denote $g\left(x^{+}\right)=\lim _{y>x, y \rightarrow x} g(y)$ and $g\left(x^{-}\right)=\lim _{y<x, y \rightarrow x} g(y)$. $\varepsilon$ is a strictly positive number that is arbitrarily close to zero. $\alpha$ is a number that is lower than and arbitrarily close to 1 .

I define some parameters that will be used along the proof. First denote $H_{1} \equiv\left\{r \in[1 / 2,1] \mid R_{1}(r)=0\right\}$ and $H_{2} \equiv\left\{r \in[1 / 2,1] \mid R_{2}(r)=0\right\}$. If $R_{1}(\cdot)>0$ in the whole domain, we could define a feasible alternative reduced-form allocation $\bar{Q}$ satisfying $\bar{Q}_{2}(\cdot)=Q_{2}(\cdot)$ and $\bar{Q}_{1}(\cdot)=Q_{1}(\cdot)+\varepsilon$, obtaining higher ex-ante welfare while maintaining BIC, and we would be done. Thus from now on I assume that $H_{1}$ is not empty. $H_{2}$ is not empty either since by construction $R_{2}(1)=0$. I define $h_{1} \equiv \inf H_{1}(>1 / 2), \hat{h}_{1} \equiv \sup H_{1}$ and $h_{2} \equiv \inf H_{2}$. Given point 4) in lemma 2, and since both $R_{1}(\cdot)$ and $R_{2}(\cdot)$ are continuous, there exists a unique point $r^{\prime} \in(1 / 2,1]$ such that $R_{1}\left(r^{\prime}\right)=R_{2}\left(r^{\prime}\right), R_{1}(r)<R_{2}(r)$ for every $r<r^{\prime}$, and $R_{1}(r)>R_{2}(r)$ for every $r>r^{\prime}$. Consequently, $\hat{h}_{1} \leq r^{\prime} \leq h_{2}$.

There are other useful parameters that I define here. $h_{3}$ (if it exists) is a point in the interval $\left[\hat{h}_{1}, h_{2}\right]$ where $Q(\cdot)$ is discontinuous. $\hat{h}_{3}$ is a number higher than and arbitrarily close to $\hat{h}_{1} . h_{4}$ is the infimum of the set $\left\{x \in\left(\hat{h}_{3}, h_{2}\right): Q_{2}\left(h_{4}\right)=\bar{Q}_{2}\left(\hat{h}_{3}\right)\right\}$ if such a set exists. The parameters $h_{1}, \hat{h}_{1}, h_{2}, h_{3}, \hat{h}_{3}, h_{4}$ will serve to find the aforementioned alternative reduced-form allocation $\bar{Q}$.

Preliminarily, I deal with the simple case in which $Q$ is constant over the whole domain $[1 / 2,1]$. Since $H_{1} \neq \varnothing$, we have $Q_{1}(\cdot)>0$ (otherwise, for any $r$ in the domain, $\left.R_{1}(r)=R_{2}(1 / 2)+(1 / 2)^{n}-[1-\Psi(r)]^{n}>0\right)$. It is also clear that $Q_{2}(\cdot)<1$ in order to preserve feasibility. I can then construct an alternative allocation $\bar{Q}$ such that $\bar{Q}_{2}(\cdot)=Q_{2}(\cdot)+\varepsilon, \bar{Q}_{1}(\cdot)=Q_{1}(\cdot)-\varepsilon$. The new reduced-form allocation is feasible, incentive compatible and welfare-improving. The net welfare gain is $\int_{1 / 2}^{1} \psi(r) \varepsilon\left[E\left[v_{2} \mid r\right]-E\left[v_{1} \mid r\right]\right] d r$, which is strictly positive, and we are done. So from now on, I will assume that $Q$ is not constant over the whole domain.

The remaining scenarios are divided into four cases. Case 1 contains with scenarios where $h_{2}=1$ whereas the other cases assume $h_{2}<1$. In case 2 , there is a discontinuity point $h_{3}$ as previously defined. In the remaining cases, such a number $h_{3}$ does not exist. In case $3, \hat{h}_{1}<h_{2}$, while in case $4, \hat{h}_{1}=h_{2}$. I extensively analyze case 1 in detail. The remaining cases are treated more concisely insofar as many of its elements are analogous to the ones of case 1.

Case 1: $h_{2}=1$.

Construction of $\bar{Q}$

(note: if $h_{1}=1$, in what follows replace $h_{1}^{+}$with 1 )

1) For $r \in\left[1 / 2, h_{1}\right], \bar{Q}_{1}(r)-Q_{1}\left(h_{1}^{+}\right)=\alpha\left[Q_{1}(r)-Q_{1}\left(h_{1}^{+}\right)\right], \bar{Q}_{2}\left(h_{1}\right)=Q_{2}\left(h_{1}^{+}\right)+\varepsilon(\varepsilon$ is set to obtain 
$\left.\bar{R}_{1}\left(h_{1}\right)=R_{1}\left(h_{1}\right)\right)$ and $\bar{Q}_{2}\left(h_{1}\right)-\bar{Q}_{2}(r)=\alpha\left[Q_{2}\left(h_{1}^{+}\right)-Q_{2}(r)\right]$

2) For $r \in\left(h_{1}, 1\right]$ (if such an interval is not empty), $\bar{Q}_{1}(r)=Q_{1}\left(r^{-}\right)$and $\bar{Q}_{2}(r)=Q_{2}\left(r^{-}\right)+\varepsilon$

Feasibility

Notice that $R_{1}(1) \geq 0$ and the fact that $Q_{1}(\cdot)+Q_{2}(\cdot)$ is nonincreasing (strictly decreasing at some point) implies that $Q_{1}(1)+Q_{2}(1)<2 / n$. Therefore $Q_{2}(1)<1$ and the constructed $\bar{Q}_{2}$ 's are proper probabilities here as well as in the remaining cases. $\bar{R}_{1}\left(h_{1}\right)=0$ and since $\bar{Q}_{1}(r)=Q_{1}\left(r^{-}\right)$for $r \in\left(h_{1}, 1\right]$, it follows that $\bar{R}_{1}(r) \geq 0$ for $r \in\left(h_{1}, 1\right]$. Since $\varepsilon$ is arbitrarily small and $\alpha$ is arbitrarily close to 1 , and since $R_{1}(r)>0$ for $r \in\left[1 / 2, h_{1}\right]$ and $R_{2}(r)>0$ for $r \in[1 / 2,1)$, we also obtain $\bar{R}_{1}(r) \geq 0$ for $r \in\left[1 / 2, h_{1}\right]$ and $\bar{R}_{2}(r) \geq 0$ for $r \in[1 / 2,1]$.

Incentive compatibility

By BIC, we have, for any $1 / 2 \leq r<h_{1}, u(r) / r-u\left(h_{1}\right) / h_{1}=\int_{r}^{h_{1}} \frac{Q_{1}(x)}{x^{2}} d x$. I show that the same equation holds regarding $\bar{u}(\cdot)$ and $\bar{Q}(\cdot)$. By construction of $\bar{Q}(\cdot)$, we have $\bar{Q}_{1}(r) \frac{1-r}{r}-Q_{1}\left(h_{1}^{+}\right) \frac{1-h_{1}}{h_{1}}=$ $\frac{1-r}{r} \alpha\left[Q_{1}(r)-Q_{1}\left(h_{1}^{+}\right)\right]+Q_{1}\left(h_{1}^{+}\right)\left(\frac{1-r}{r}-\frac{1-h_{1}}{h_{1}}\right)$ and $\bar{Q}_{2}\left(h_{1}\right)-\bar{Q}_{2}(r)=\alpha\left[Q_{2}\left(h_{1}^{+}\right)-Q_{2}(r)\right]$. Substracting the second equation from the first, I obtain:

$$
\begin{aligned}
\bar{u}(r) / r-\bar{u}\left(h_{1}\right) / h_{1} & =\alpha\left[u(r) / r-u\left(h_{1}\right) / h_{1}\right]+(1-\alpha) Q_{1}\left(h_{1}^{+}\right)\left(\frac{1-r}{r}-\frac{1-h_{1}}{h_{1}}\right) \\
& =\int_{r}^{h_{1}} \frac{\alpha Q_{1}(x)}{x^{2}} d x+(1-\alpha) Q_{1}\left(h_{1}^{+}\right)\left(\frac{1-r}{r}-\frac{1-h_{1}}{h_{1}}\right) \\
& =\int_{r}^{h_{1}} \frac{\bar{Q}_{1}(x)-(1-\alpha) Q_{1}\left(h_{1}^{+}\right)}{x^{2}} d x+(1-\alpha) Q_{1}\left(h_{1}^{+}\right)\left(\frac{1-r}{r}-\frac{1-h_{1}}{h_{1}}\right) \\
& =\int_{r}^{h_{1}} \frac{\bar{Q}_{1}(x)}{x^{2}} d x
\end{aligned}
$$

For any other value $r \geq h_{1}$, the function $\bar{Q}_{2}(\cdot)$ is just a swifting-up of $Q_{2}(\cdot)$, thus it is easy to see that the condition $\bar{u}\left(h_{1}\right) / h_{1}-\bar{u}(r) / r=\int_{h_{1}}^{r} \frac{\bar{Q}_{1}(x)}{x^{2}} d x$ holds. It is consequently clear that for any pair $(r, \tilde{r})$ the equation $\bar{u}(\tilde{r}) / \tilde{r}-\bar{u}(r) / r=\int_{\tilde{r}}^{r} \frac{\bar{Q}_{1}(x)}{x^{2}} d x$ holds, while $\bar{Q}_{2}(\cdot)$ is nondecreasing (or $\bar{Q}_{1}(\cdot)$ is nonincreasing) in the whole domain. Invoking lemma $3, \bar{Q}(\cdot)$ preserves BIC.

Welfare improvement

I show that $\varepsilon$ is a strictly positive number as required. As stated, $\varepsilon$ is set to keep $\bar{R}_{1}\left(h_{1}\right)=R_{1}\left(h_{1}\right)$, that is,

$$
\begin{aligned}
& 1-\left[1-\Psi\left(h_{1}\right)\right]^{n}-n \int_{h_{1}}^{1} \psi(x) \bar{Q}_{2}(x) d x-n \int_{1 / 2}^{h_{1}} \psi(x)\left[\bar{Q}_{1}(x)+\bar{Q}_{2}(x)\right] d x \\
= & 1-\left[1-\Psi\left(h_{1}\right)\right]^{n}-n \int_{h_{1}}^{1} \psi(x) Q_{2}(x) d x-n \int_{1 / 2}^{h_{1}} \psi(x)\left[Q_{1}(x)+Q_{2}(x)\right] d x
\end{aligned}
$$


leading to

$$
\begin{aligned}
& 1-\left[1-\Psi\left(h_{1}\right)\right]^{n}-n \int_{h_{1}}^{1} \psi(x) Q_{2}(x) d x-n\left[Q_{1}\left(h_{1}^{+}\right)+Q_{2}\left(h_{1}^{+}\right)\right]\left[\Psi\left(h_{1}\right)-1 / 2\right] \\
& -\alpha n \int_{1 / 2}^{h_{1}} \psi(x)\left[Q_{1}(x)-Q_{1}\left(h_{1}^{+}\right)+Q_{2}(x)-Q_{2}\left(h_{1}^{+}\right)\right] d x \\
& -n \varepsilon / 2 \\
= & 1-\left[1-\Psi\left(h_{1}\right)\right]^{n}-n \int_{h_{1}}^{1} \psi(x) Q_{2}(x) d x-n\left[Q_{1}\left(h_{1}^{+}\right)+Q_{2}\left(h_{1}^{+}\right)\right]\left[\Psi\left(h_{1}\right)-1 / 2\right] \\
& -n \int_{1 / 2}^{h_{1}} \psi(x)\left[Q_{1}(x)-Q_{1}\left(h_{1}^{+}\right)+Q_{2}(x)-Q_{2}\left(h_{1}^{+}\right)\right] d x
\end{aligned}
$$

Since by BIC $Q_{1}(\cdot)+Q_{2}(\cdot)$ is nonincreasing (strictly decreasing when $Q(\cdot)$ varies), we obtain $Q_{1}(x)$ $Q_{1}\left(h_{1}^{+}\right)+Q_{2}(x)-Q_{2}\left(h_{1}^{+}\right) \geq 0$ for any $x \leq h_{1}$, strictly for a positive-measured set of $x$ 's. Should $Q(\cdot)$ be constant along the interval between $1 / 2$ and $h_{1}, R_{1}\left(h_{1}\right)=0$ implies $0>R_{1}^{\prime}\left(h_{1}^{-}\right)=n\left[\left[1-\Psi\left(h_{1}\right)\right]^{n-1}-Q_{1}\left(h_{1}^{-}\right)\right]$ so $Q_{1}(\cdot)$ must jump down at $h_{1}$ (since feasibility requires $R_{1}^{\prime}\left(h_{1}^{+}\right)=n\left[\left[1-\Psi\left(h_{1}\right)\right]^{n-1}-Q_{1}\left(h_{1}^{+}\right)\right] \geq 0$ ), and then $Q_{1}(x)-Q_{1}\left(h_{1}^{+}\right)+Q_{2}(x)-Q_{2}\left(h_{1}^{+}\right)>0$ for any $x \leq h_{1}$. All the integrals in the equation above take then strictly positive value, thus $\varepsilon>0$ as required. $\varepsilon$ is arbitrarily small given that $\alpha$ is arbitrarily close to 1.

Given $\varepsilon>0$, for any $r \geq 1 / 2$, we have $\bar{Q}_{2}(r) \geq Q_{2}(r)$ (strictly for a positive-measured subset of the domain). Since $\bar{R}_{1}\left(h_{1}\right)=R_{1}\left(h_{1}\right)$, it follows that $\int_{1 / 2}^{1}\left[\bar{Q}_{2}(x)-Q_{2}(x)\right] \psi(x) d x=\int_{1 / 2}^{h_{1}}\left[Q_{1}(x)-\bar{Q}_{1}(x)\right] \psi(x) d x$. The regularity condition implies that

$$
\int_{1 / 2}^{1}\left[\bar{Q}_{2}(x)-Q_{2}(x)\right] E\left(v_{2} \mid x\right) \psi(x) d x>\int_{1 / 2}^{1}\left[Q_{1}(x)-\bar{Q}_{1}(x)\right] E\left(v_{1} \mid x\right) \psi(x) d x
$$

so that $\bar{Q}$ induces a net ex-ante welfare gain with respect to $Q$.

Case 2: $h_{2}<1$, and there is $h_{3} \in\left[\hat{h}_{1}, h_{2}\right]$ such that $Q(\cdot)$ is discontinuous at $h_{3}$.

Construction of $\bar{Q}$

1) For $r \in\left[1 / 2, h_{1}\right], \bar{Q}_{1}(r)-Q_{1}\left(h_{1}^{+}\right)=\alpha\left[Q_{1}(r)-Q_{1}\left(h_{1}^{+}\right)\right], \bar{Q}_{2}\left(h_{1}\right)=Q_{2}\left(h_{1}^{+}\right)+\varepsilon(\varepsilon$ is set to obtain $\left.\bar{R}_{1}\left(h_{1}\right)=R_{1}\left(h_{1}\right)\right)$ and $\bar{Q}_{2}\left(h_{1}\right)-\bar{Q}_{2}(r)=\alpha\left[Q_{2}\left(h_{1}^{+}\right)-Q_{2}(r)\right]$

2) For $r \in\left(h_{1}, h_{3}\right]$ (if such an interval is not empty), $\bar{Q}_{1}(r)=Q_{1}\left(r^{-}\right)$and $\bar{Q}_{2}(r)=Q_{2}\left(r^{-}\right)+\varepsilon$

3) For $r \in\left(h_{3}, 1\right], \bar{Q}_{1}(r)=Q_{1}\left(r^{-}\right)+\frac{h_{3}}{1-h_{3}} \varepsilon$, and $\bar{Q}_{2}(r)=Q_{2}\left(r^{-}\right)$.

Feasibility

The only additional issue that is worth to mention here, with respect to case 1 , is that although $\bar{Q}_{1}(r)>$ $Q_{1}\left(r^{-}\right)$for all $r \in\left(h_{3}, 1\right]$, this does not violate feasibility since $\varepsilon$ is sufficiently small and $R_{1}(r)>0$ for all $r \in\left(\hat{h}_{1}, 1\right]$.

Incentive compatibility 
It works as in case 1 once again. It is just necessary to additionally consider BIC around $h_{3}$. That requires $\bar{u}(\cdot)$ to be continuous at $h_{3}$. Since $\bar{u}\left(h_{3}^{-}\right)=h_{3}\left[Q_{2}\left(h_{3}^{-}\right)+\varepsilon\right]+\left(1-h_{3}\right) Q_{1}\left(h_{3}^{-}\right)$and $\bar{u}\left(h_{3}^{+}\right)=h_{3} Q_{2}\left(h_{3}^{-}\right)+(1-$ $\left.h_{3}\right)\left[Q_{1}\left(h_{3}^{-}\right)+\frac{h_{3}}{1-h_{3}} \varepsilon\right]$, it is clear that $\bar{u}\left(h_{3}^{-}\right)=\bar{u}\left(h_{3}^{+}\right)=\bar{u}\left(h_{3}\right)$.

Welfare improvement

Analogously to case 1 , it is easy to check that $\varepsilon>0$ as required. As in case 1 , since $\bar{R}_{1}\left(h_{1}\right)=R_{1}\left(h_{1}\right)$, and given the regularity condition, the welfare gain given by the increase in $Q_{2}$ is higher than the welfare loss induced by the decrease in $Q_{1}$. Moreover, $Q_{1}$ is increased in the interval $\left(h_{3}, 1\right]$.

Case 3: $\hat{h}_{1}<h_{2}<1$, and there is no $h_{3} \in\left[\hat{h}_{1}, h_{2}\right]$ such that $Q(\cdot)$ is discontinuous at $h_{3}$.

Construction of $\bar{Q}$

1) For $r \in\left[1 / 2, h_{1}\right], \bar{Q}_{1}(r)-Q_{1}\left(h_{1}^{+}\right)=\alpha\left[Q_{1}(r)-Q_{1}\left(h_{1}^{+}\right)\right], \bar{Q}_{2}\left(h_{1}\right)=Q_{2}\left(h_{1}^{+}\right)+\varepsilon(\varepsilon$ is set to obtain $\left.\bar{R}_{1}\left(h_{1}\right)=R_{1}\left(h_{1}\right)\right)$ and $\bar{Q}_{2}\left(h_{1}\right)-\bar{Q}_{2}(r)=\alpha\left[Q_{2}\left(h_{1}^{+}\right)-Q_{2}(r)\right]$

2) For $r \in\left(h_{1}, \hat{h}_{3}\right], \bar{Q}_{1}(r)=Q_{1}\left(r^{-}\right)$and $\bar{Q}_{2}(r)=Q_{2}\left(r^{-}\right)+\varepsilon$

3) For $r \in\left(\hat{h}_{3}, h_{4}\right], \bar{Q}(r)=\bar{Q}\left(\hat{h}_{3}\right)$

4) For $r \in\left(h_{4}, 1\right], \bar{Q}_{2}(r)=Q_{2}(r)$ and $\bar{Q}_{1}(r)-Q_{1}(r)=\bar{Q}_{1}\left(h_{4}\right)-Q_{1}\left(h_{4}\right)$

Feasibility

First of all, given that $\hat{h}_{3}$ is sufficiently close to $\hat{h}_{1}, h_{4}$ exists because $Q_{2}(\cdot)$ cannot be constant around $h_{2}$ (since $R_{2}\left(h_{2}\right)=0$ and $R_{2}$ is differentiable for $r$ lower than and close to $h_{2}$, with $R_{2}^{\prime}(r)=-n\left[\Psi(r)^{n-1}-Q_{2}(r)\right]$, $R_{2}^{\prime}(r) \leq 0=R_{2}^{\prime}\left(h_{2}\right)$ implies $\left.Q_{2}(r)<Q_{2}\left(h_{2}\right)\right)$.

Being $\varepsilon$ small enough and $\hat{h}_{3}$ is sufficiently close to $\hat{h}_{1}$, the difference $\bar{Q}_{1}(r)-Q_{1}(r)$ for $r \in\left(h_{4}, 1\right]$ is also small enough to preserve $\bar{R}_{1}(\cdot) \geq 0$ along the interval $\left[\hat{h}_{3}, 1\right]$ (where $R_{1}$ is strictly positive). Keeping $\bar{Q}_{1}(r)=Q_{1}\left(r^{-}\right)$in the short interval $\left(\hat{h}_{1}, \hat{h}_{3}\right]$ serves to avoid feasibility problems derived from $R_{1}\left(\hat{h}_{1}\right)=0$.

Incentive compatibility

That there are no incentive compatibility problems in the interval $\left[1 / 2, h_{1}\right]$ is again seen along the lines of case 1. In the remaining intervals, the new allocation function $\bar{Q}$ is either a swift of $Q$ or a constant function, with no discontinuities in the $\left(\hat{h}_{1}, h_{2}\right)$ interval. Therefore BIC is preserved given that $Q$ is Bayesian incentive compatible.

Welfare improvement

The fact that $\varepsilon>0$ can be checked with minor changes with respect to case 1 . By the regularity condition, the welfare gain given by the increase in $Q_{2}$ is higher than the welfare loss induced by the decrease in $Q_{1}$. Moreover, $Q_{1}$ is increased in the interval $\left(\hat{h}_{3}, 1\right]$.

Case 4: $\hat{h}_{1}=h_{2}<1$.

In this last case, I show that there must be a discontinuity in $Q(\cdot)$ at $h_{2}$, leading to case 2 . By the facts 
that $R_{1}\left(h_{2}\right)-R_{2}\left(h_{2}\right)=0$ and $Q_{1}(\cdot)+Q_{2}(\cdot)$ is nonincreasing one obtains

$$
\begin{aligned}
& \Psi\left(h_{1}\right)^{n}-\left[1-\Psi\left(h_{1}\right)\right]^{n} \\
= & n \int_{1 / 2}^{h_{2}} \psi(x)\left[Q_{1}(x)+Q_{2}(x)\right] d x \\
\geq & n\left[\Psi\left(h_{2}\right)-1 / 2\right]\left[Q_{1}\left(h_{2}\right)+Q_{2}\left(h_{2}\right)\right]
\end{aligned}
$$

After defining $M(x) \equiv \frac{x^{n}-\left[1-\Psi\left(h_{2}\right)\right]^{n}}{n\left[\Psi\left(h_{2}\right)-x\right]}+\frac{\Psi\left(h_{2}\right)^{n}-x^{n}}{n\left[\Psi\left(h_{2}\right)-x\right]}, x \in\left[1 / 2, \Psi\left(h_{2}\right)\right)$, I observe that $Q_{1}\left(h_{2}\right)+Q_{2}\left(h_{2}\right) \leq$ $M(1 / 2)$. On the other hand, $R_{2}\left(h_{2}\right)=0$ implies $Q_{2}\left(h_{2}^{+}\right) \geq \Psi\left(h_{2}\right)^{n-1}\left(\right.$ since $\left.R_{2}^{\prime}\left(h_{2}^{+}\right) \geq 0\right)$ and $R_{1}\left(h_{2}\right)=0$ implies $Q_{1}\left(h_{2}^{-}\right) \geq\left[1-\Psi\left(h_{2}\right)\right]^{n-1}$ (since $\left.R_{1}^{\prime}\left(h_{2}^{-}\right) \leq 0\right)$. If $Q$ were continuous at $h_{2}$, we would have $Q_{1}\left(h_{2}\right)+$ $Q_{2}\left(h_{2}\right) \geq\left[1-\Psi\left(h_{2}\right)\right]^{n-1}+\Psi\left(h_{2}\right)^{n-1}=M\left(\Psi\left(h_{2}\right)^{-}\right)$. Since $M(x)$ is strictly increasing in $x \in\left[1 / 2, \Psi\left(h_{2}\right)\right)$, by contradiction $Q$ is discontinuous at $h_{2}$. This leads to case 2 as previously stated.

\section{Proof. Derivation of equilibrium bid and price strategies $B(\cdot)$ and $P(\cdot)$ in Section 3.}

I first derive the equilibrium bid function $B(\cdot)$ in the $n$-player auction depicted in Section 3 . For a $v$-type agent, where $v=\left(v_{1}, v_{2}\right)$ is the vector of her valuations, define $m \equiv v_{1} / v_{2}$, her marginal rate of substitution. Assume that all agents prefer object 2, that is, $m \in[0,1]$. Let $G(\cdot)$ be the distribution of an agent's $m$ conditional on being in the $[0,1]$ interval, and $g(\cdot)$ be its associated density (let $\hat{m}$ demote $m$ when considered as a random variable). In the rest of this derivation, agents' types will be sufficiently characterized by the marginal rate of substitution. Given a supposedly strictly increasing and differentiable equilibrium bid function $\beta(\cdot)$, an $m$-type agent chooses her bid $b$ (in probability units of obtaining object 2) in order to maximize her expected payoff

$$
\pi(m ; b)=G\left(\beta^{-1}(b)\right)^{n-1}(m-b)+\frac{1}{n-1} \int_{\beta^{-1}(b)}^{1} \beta(\mu) d G(\mu)^{n-1}
$$

where the first component of this sum is the usual one in first-price auctions and the second component comes from the fact that the winning bid is evenly split among the losers in case the agent does not win the auction. By the envelope theorem we know that $\frac{d \pi}{d m}=\frac{\partial \pi}{\partial m}=G\left(\beta^{-1}(b)\right)^{n-1}$. By the symmetry of the equilibrium, we know that $b=\beta(m)$. Thus we have

$$
\pi(m ; \beta(m))=\pi(0 ; \beta(0))+\int_{0}^{m} G(\mu)^{n-1} d \mu
$$

where $\pi(0 ; \beta(0))=\frac{1}{n-1} \int_{0}^{1} \beta(\mu) d G(\mu)^{n-1}$. I combine the two previously displayed equations to obtain

$$
G(m)^{n-1} m-\int_{0}^{m} G(\mu)^{n-1} d \mu=G(m)^{n-1} \beta(m)+\frac{1}{n-1} \int_{0}^{m} \beta(\mu) d G(\mu)^{n-1}
$$

which holds for every $m \in[0,1]$. I differentiate the equation and I multiply it by $(n-1) G(m)$ obtaining

$$
\frac{n-1}{n} m n g(m) G(m)^{n-1}=n g(m) G(m)^{n-1} \beta(m)+G(m)^{n} \beta^{\prime}(m)
$$


where the right hand side is equivalent to $\frac{d\left[G(m)^{n} \beta(m)\right]}{d m}$. Integrating I solve for $\beta(m)$ :

$$
\beta(m)=\frac{n-1}{n G(m)^{n}} \int_{0}^{m} \mu d G(\mu)^{n}=\frac{n-1}{n} E_{N}\left(\max _{i \in N} \hat{m}^{i} \mid \max _{i \in N} \hat{m}^{i}<m\right)
$$

$B(r)$ equals $\beta\left(\frac{1-r}{r}\right)$, for any $r \geq 1 / 2$. An analogous derivation follows for the shape of $B(\cdot)$ when all agents prefer object 1 . Obviously, the same derivation applies when $n=2$.

I derive $P(\cdot)$ for such a case in which we have two agents. I newly assume that both agents prefer the same object, say object 2 . I assume that $P(\cdot)$ is decreasing in $r$. Then, by the rules of the designed market, the agent with the most moderate preference (say $r$ ) obtains the non-preferred object, in exchange of $\frac{1-P(r)}{2 P(r)}$ in probability units of object 2 . This payment is received by the agent who loses the non-preferred object. Each agent's strategy could be redefined in terms of $\pi(r) \equiv \frac{1-P(\cdot)}{2 P(\cdot)}$, and it is easy to see that this game is isomorphic with respect to the auction game for which I just derived the symmetric equilibrium. In the symmetric equilibrium of the market game I obtain $\pi(r)=B(r)$ for any $r \geq 1 / 2$.

\section{Proof. Propositions 3 and 4.}

For the purposes of this proof, consider the following uninsured auction mechanism. Each agent $i$ reports her preferred object $h^{i} \in\{1,2\}$ and a bid $b^{i} \in[0,1]$ to the planner in a sealed envelope. If not all the agents declare to prefer the same object, each object is randomly assigned to an agent who reports that she prefers it. If all agents declare to prefer the same object $h$, then: 1) an endowment probability $1 / n$ of obtaining that object is given to each agent, and 2) the other object $3-h$ is auctioned off and given to the agent with the highest (sealed) bid, who pays her bid in probability units of obtaining object $h$, and this payment is evenly transferred to the agents who lose the auction (they do not pay their bids). In this uninsured auction mechanism, we ignore the fact that the bids might be capped by the endowment, that is, negative allocation probabilities for good $h$ might occur.

The proof works as follows. I first show that this uninsured auction mechanism generates the same interim allocation probabilities as the optimal mechanism. This is relatively easy given the Revenue Equivalence Theorem. This would complete the proof of proposition 3 because the equilibrium bid never exceeds the endowment $1 / n$ when $n=2$. The problem when $n>2$ is that the uninsured auction mechanism may violate the feasibility constraints (it may assign negative probabilities). I then show that the insured auction mechanism depicted in Section 3, which is feasible, implements the same interim allocation probabilities as the uninsured auction mechanism. This would complete the proof of proposition 4.

In both the insured and uninsured auction mechanism agents truthfully declare their ordinal preferences as part of a symmetric equilibrium where the bid function meets $B(r)=B(1-r)$ for any $r$. For any given bid, an agent who deviates and lies about her ordinal preferences would obtain higher interim chances to obtain the object she does not prefer and lower for the object she prefers. 
Ignoring the endowment constraint as assumed, the symmetric equilibrium bid strategy in the uninsured auction mechanism is $B(r)=\frac{n-1}{n} E_{N}\left(\max _{i \in N} \frac{1-\hat{r}^{i}}{\hat{r}^{i}} \mid \frac{1-r}{r}>\max _{i \in N} \frac{1-\hat{r}^{i}}{\hat{r}^{i}}\right)$ for $r \geq 1 / 2$ and $B(r)=\frac{n-1}{n} E_{N}$ $\left(\max _{i \in N} \frac{\hat{r}^{i}}{1-\hat{r}^{i}} \mid \frac{r}{1-r}>\max _{i \in N} \frac{\hat{r}^{i}}{1-\hat{r}^{i}}\right)$ for $r<1 / 2$ (observe that $B(r)=B(1-r)$ for any $r$ ). This has been derived in the previous proof. We focus on agents of type $r \geq 1 / 2$. In case all agents prefer object 2 , and since $B(r)$ is strictly decreasing in $r$, object 1 is allocated for sure to the agent with the lowest preference intensity (lowest $r$ ). And if some other agent prefers object 1 instead, the $r$-type agent has no chance to obtain object 1. Therefore $Q_{1}^{U N A U C}(r)=[1-\Psi(r)]^{n-1}$, where the $U N A U C$ superscript stands for the symmetric equilibrium in the uninsured auction mechanism. When $r=1 / 2$, this type's bid is the winning bid with probability 1 in case there is an auction. Hence this agent's interim expected payment consists of her bid $B(1 / 2)$, thus $Q_{2}^{U N A U C}(1 / 2)=\sum_{\tilde{n}=0}^{n-2}(1 / 2)^{n-1}\left(\begin{array}{c}n-1 \\ \tilde{n}\end{array}\right) \frac{1}{\tilde{n}+1}+(1 / 2)^{n-1}[1 / n-B(1 / 2)]$. The first component $\sum_{\tilde{n}=0}^{n-2}(1 / 2)^{n-1}\left(\begin{array}{c}n-1 \\ \tilde{n}\end{array}\right) \frac{1}{\tilde{n}+1}$ consider states of the world in which not all agents prefer object 2 (and object 2 is randomly assigned among those agents who prefer it). In the second element, $(1 / 2)^{n-1}$ is the probability that everyone else prefers object 2 (thus object 1 is auctioned off and the agent with type $r=1 / 2$ wins and pays her bid).

Considering the optimal mechanism, the corollary of proposition 2 establishes $Q_{1}^{*}(r)=[1-\Psi(r)]^{n-1}=$ $Q_{1}^{U N A U C}(r), r \in[1 / 2,1]$, where the star superscript identifies the optimal mechanism. This function is differentiable in the interior of the domain. Thus $Q_{2}^{*}(\cdot)$ is differentiable almost everywhere, and by equation (1) $Q_{2}^{* \prime}(r)=-\frac{1-r}{r} Q_{1}^{* \prime}(r)=\frac{1-r}{r}(n-1) \psi(r)[1-\Psi(r)]^{n-2}$. I use the result in proposition $1, R_{2}(1 / 2)=0$, to calculate $Q_{2}^{*}(1 / 2)$.

$$
\begin{aligned}
& R_{2}(1 / 2)=1-(1 / 2)^{n}-n \int_{1 / 2}^{1} \psi(r)\left[Q_{2}^{*}(1 / 2)+\int_{1 / 2}^{r} \frac{1-x}{x}(n-1) \psi(x)[1-\Psi(x)]^{n-2} d x\right] d r=0 \text { gives } \\
& Q_{2}^{*}(1 / 2)=\frac{1-(1 / 2)^{n}}{n / 2}-(1 / 2)^{n-1} \frac{n-1}{n} \int_{1 / 2}^{1} \frac{1-r}{r} n \frac{\psi(r)}{1 / 2}\left[\frac{1-\Psi(r)}{1 / 2}\right]^{n-1} d r \\
&=\frac{1-(1 / 2)^{n}}{n / 2}-(1 / 2)^{n-1} \frac{n-1}{n} E_{N}\left(\max _{i \in N} \frac{1-\tilde{r}^{i}}{\tilde{r}^{i}} \mid 1 / 2<\tilde{r}^{1}, \ldots, 1 / 2<\tilde{r}^{n}\right) \\
&=\sum_{\tilde{n}=0}^{n-1}(1 / 2)^{n-1}\left(\begin{array}{c}
n-1 \\
\tilde{n}
\end{array}\right) \frac{1}{\tilde{n}+1}-(1 / 2)^{n-1} B(1 / 2) \\
&=\sum_{\tilde{n}=0}^{n-2}(1 / 2)^{n-1}\left(\begin{array}{c}
n-1 \\
\tilde{n}
\end{array}\right) \frac{1}{\tilde{n}+1}+(1 / 2)^{n-1}[1 / n-B(1 / 2)]=Q_{2}^{U N A U C}(1 / 2)
\end{aligned}
$$

Since $Q_{1}^{*}(r)=Q_{1}^{U N A U C}(r), r \in[1 / 2,1]$ and $Q_{2}^{*}(1 / 2)=Q_{2}^{U N A U C}(1 / 2)$, the revenue equivalence theorem implies that $Q_{2}^{*}(r)=Q_{2}^{U N A U C}(r), r \in[1 / 2,1]$. A symmetric argument follows when $r<1 / 2$. Thus the uninsured auction mechanism implements the optimal mechanism provided it allocates positive probabilities.

The previous proof in the appendix showed that the market mechanism and the (uninsured) auction mechanism where isomorphic when $n=2$. And when $n=2, B(\cdot)$ does never exceed the endowment $1 / 2$ (i.e. 
the uninsured auction mechanism always provides feasible random allocations). All this proves proposition 3.

To continue with proposition 4, I only need to show that the insured auction mechanism implements the same interim allocation probabilities as the uninsured auction mechanism. For $r \geq 1 / 2$ (a symmetric argument would follow for $r<1 / 2$ ), it is clear that both mechanisms implement the same interim probabilities with respect to object 1, the non-preferred object. Yet the insurance policy in the insured auction mechanism may alter the interim allocation probabilities of object 2, the preferred object, as compared to those of the uninsured auction mechanism. I assume that the bid function $B(\cdot)$ is also a symmetric equilibrium bid function in the insured auction mechanism. I check that under this assumption the interim probabilities for object 2 are the same in both the insured and the uninsured auction mechanisms. Thus both mechanisms implement the same interim probabilities in both objects, coinciding with the interim probabilities of the optimal incentive-compatible mechanism. This also confirms that the assumed bid function $B(\cdot)$ is also an equilibrium bid function in the insured auction mechanism.

Let $Q_{2}^{I N A U C}(r)$ denote the interim allocation probability for object 2 to an $r$-type agent playing the equilibrium bid strategy $B(\cdot)$ in the insured auction mechanism. The difference $Q_{2}^{I N A U C}(r)-Q_{2}^{U N A U C}(r)$ is the sum of four elements:

a) The voucher $V(B(r))$ received when object 1 is auctioned off and the agent wins the object, an event that happens with probability $(1 / 2)^{n-1}\left[\frac{1-\Psi(r)}{1 / 2}\right]^{n-1}$

b) The payment $\frac{1-\Psi(r)}{(n-1) / 2} V(B(r))$ made when all but one of the other agents prefer object 2 and $r$ is the lowest preference intensity among them, an event that happens with probability $(n-1)(1 / 2)^{n-1}\left[\frac{1-\Psi(r)}{1 / 2}\right]^{n-2}$

c) The expected voucher that is not paid by the winner of the auction (different from the agent) when all agents prefer object $2,(1 / 2)^{n-1} \int_{1 / 2}^{r} \frac{V(B(x))}{n-1} \cdot(n-1) \frac{\psi(x)}{1 / 2}\left[\frac{1-\Psi(x)}{1 / 2}\right]^{n-2} d x$

d) The expected voucher that another agent pays when there are $n-1$ agents preferring object 2 , and this agent has the lowest preference intensity, $(n-1)(1 / 2)^{n-1} \int_{1 / 2}^{r} \frac{1-\Psi(x)}{(n-1) / 2} \frac{V(B(x))}{n-2} \cdot(n-2) \frac{\psi(x)}{1 / 2}\left[\frac{1-\Psi(x)}{1 / 2}\right]^{n-3} d x$

It is easy to see that a) and b) cancel each other, and c) and d) cancel each other. Therefore $Q_{2}^{I N A U C}(r)=$ $Q_{2}^{U N A U C}(r)$. 